\title{
Dynamic synthetic cells based on liquid-liquid phase separation
}

\author{
Nicolas Martin, ${ }^{*[a]}$ \\ [a] Univ. Bordeaux, CNRS, Centre de Recherche Paul Pascal, UMR5031, 115 Avenue du Dr. Albert Schweitzer, \\ F-33600 Pessac, France
}

\begin{abstract}
"This is the accepted version of the following article: N. Martin, ChemBioChem, 2019, 20, 2553-2568, which has been published in final form at http://dx.doi.org/10.1002/cbic.201900183. This article may be used for non-commercial purposes in accordance with Wiley-VCH Terms and Conditions for Self-Achiving."
\end{abstract}

\begin{abstract}
Living cells have long been a source of inspiration for chemists. Their capacity of performing complex tasks relies on the spatiotemporal coordination of matter and energy fluxes. Recent years have witnessed growing interest in the bottom-up construction of cell-like models capable of reproducing aspects of such dynamic organisation. Liquid--liquid phase-separation (LLPS) processes in water are increasingly recognised as a viable compartmentalisation strategy through which to produce dynamic synthetic cells. Herein, we highlight examples of the dynamic properties of LLPS used to assemble synthetic cells, including their biocatalytic activity, reversible condensation and dissolution, growth and division, and recent directions towards the design of higher-order structures and behaviour.
\end{abstract}

\section{Introduction}

One of the most fascinating hallmarks of living cells is their unique ability to coordinate matter exchanges and energy fluxes dynamically in space and time. This property, as yet unmatched in synthetic assemblies, allows them to perform complex functions.

Reproducing such dynamic organisation in artificial systems is now attracting growing interest in the contexts of fabrication of smart materials with life-inspired functionality, ${ }^{[1,2]}$ including programmability and autonomy, sensing, information processing, replication or collective coordination. It also provides new opportunities to shed light on the transition from non-living to living matter and to unveil the physicochemical bases of life. ${ }^{[3]}$ Crucial to the dynamic organisation of living matter is compartmentalisation, which allows localisation and positional assembly of functional components, confinement of incompatible chemical species, and establishment and maintenance of biochemical gradients.

Beyond the canonical membrane-bound intracellular organelles, it has become increasingly recognised in recent years that living cells also organise their contents through liquid--liquid phase-separation (LLPS) phenomena that produce membrane-free organelles, also known as biomolecular condensates. ${ }^{[4]}$ Significantly, these liquid-like droplets of proteins and RNA exhibit very rich dynamics from the nanoscale to the microscale. ${ }^{[5]}$ Their lack of membranes favours molecular exchange with their environment, whereas their liquid-like nature promotes intradroplet molecular diffusion, hence supporting localised biochemical reactions. At the microscale, the assembly and dissolution of such droplets is tightly regulated in response to environment cues, and the droplets exhibit behaviour typical of liquids, including flow under shear and fusion. Conversely, changes in the physical properties of the droplets can lead to arrested dynamics and favour protein aggregation, which has been related to possible diseases.

Simultaneously, membrane-free microdroplets formed through aqueous LLPS have emerged as representing promising compartmentalisation strategies through which to assemble synthetic cells (sometimes also referred to as protocells, or artificial cells). Such droplets provide a viable means to generate chemically enriched environments, thereby reproducing aspects of the crowded interiors of cells, and ensure facile exchanges of matter with their surroundings, hence acting as "open" reactors capable of selective sequestration of biomolecules and catalytic activity. The design of dynamic LLPS-based systems is a vibrant research area in the synthetic cell community. This review highlights examples of the dynamic properties of LLPS used to assemble synthetic cell models, including their biocatalytic activity, reversible condensation and dissolution, growth and division, and recent directions towards the design of higher-order structures and behaviour.

\section{Types of aqueous liquid-liquid phase separation used in synthetic cells}

It is well established that aqueous colloidal solutions undergo, under appropriate conditions, LLPS that is either segregative or associative ${ }^{[6]}$ (Figure 1). This section briefly highlights the main characteristics of these two phaseseparation processes.

\subsection{Segregative LLPS: synthetic cells based on aqueous two-phase systems}

"Segregative phase separation" refers to the formation of two immiscible aqueous liquid phases in equilibrium for mixtures of two (or multiple) polymers, or of a polymer and a salt, in water above threshold concentrations. ${ }^{[7]}$ In the limiting case of two segregating polymers, this phenomenon produces an aqueous two-phase system (ATPS) in which each phase is enriched in one or the other polymer (Figure 1). The term "polymer incompatibility" has conventionally been used to describe this phenomenon, although the underlying assumption of a net repulsion 
between polymers is not always accurate, because polymer--solvent interactions alone may drive phase separation.[6] One of the most commonly used classes of ATPSs involves mixtures of dextran - a natural polysaccharide - and poly(ethylene glycol) (PEG). The physics underlying phase separation through segregation of these neutral polymers in water can be qualitatively described in terms of the Flory--Huggins theory. ${ }^{[6,8]}$ Phase diagrams reveal that demixing occurs above threshold concentrations of PEG and dextran (Figure 1C) that depend on the temperature and the polymer molecular weight. The physical properties of ATPSs include low interfacial tension ${ }^{[9,10]}$ (typically between $0.5 \mu \mathrm{N} \cdot \mathrm{m}^{-1}$ and $0.5 \mathrm{mN} \cdot \mathrm{m}^{-1}$ ) and a viscosity that can easily be tuned by varying the molecular weight of the polymers. ${ }^{[11]}$

Since the seminal work in the mid-1950s by Albertsson, who demonstrated the extraction of cellular components based on differential affinity for the two phases of ATPSs, ${ }^{[7]}$ segregative phase separation has attracted strong interest in biology. Empirical observations, supported by theoretical models, have shown that biomolecules partition unevenly in the two phases while retaining their biological activity. ${ }^{[12]}$ On the basis of these favourable properties, the use of ATPSs has been extended to the assembly of rudimentary synthetic cell models. In pioneering studies, Keating and co-workers demonstrated co-encapsulation of PEG/dextran ATPSs within lipid vesicles, ${ }^{[13-15]}$ thereby reproducing aspects of cellular compartmentalisation combining a crowded (cytoplasm-like) interior delineated by a lipid bilayer. Since then, simpler models of synthetic cells, based on ATPSs involving the dispersion of one phase into the other to produce membrane-free micrometre-sized droplets capable of passive matter exchange with their surroundings, have been developed. ${ }^{[12]}$

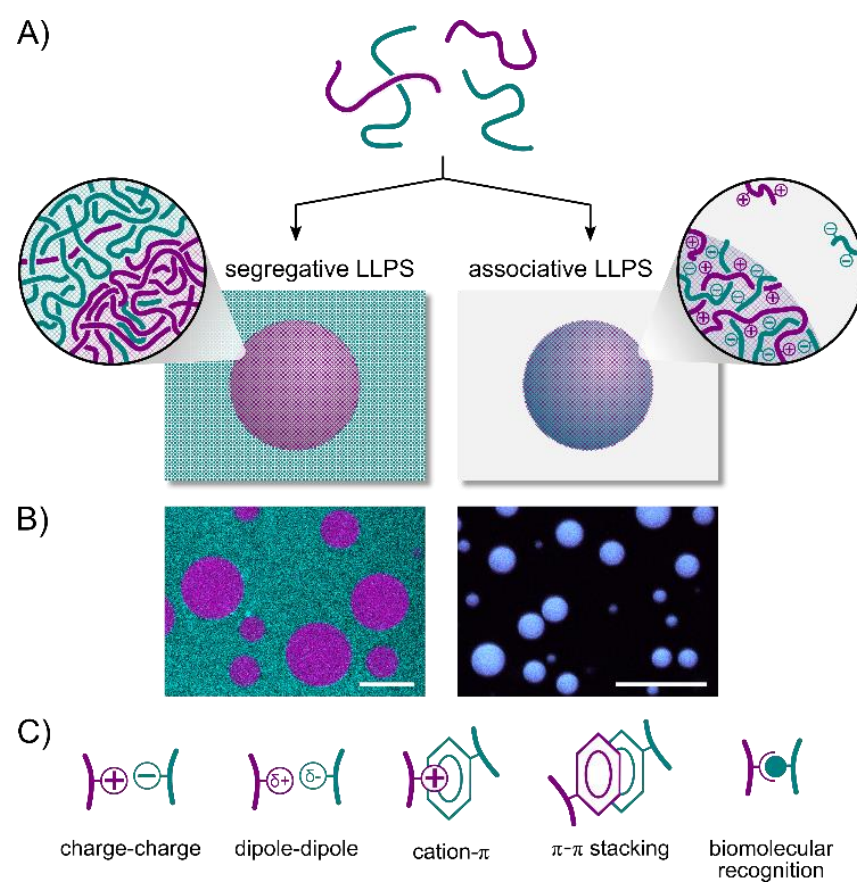

Figure 1. A) Schematic representation of segregative and associative liquid-liquid phase separation. B) Confocal fluorescence images of an aqueous two-phase system (ATPS, left) and droplets formed by complex coacervation (right). The ATPS is made of PEG and dextran doped with fluorescein isothiocyanate (FITC)-labelled PEG (cyan) and rhodamine isothiocyanate (RITC)-tagged dextran (magenta); complex coacervate droplets are assembled from polyethyleneimine (PEI) and DNA doped with FITC-tagged DNA and RITC-labelled PEI. C) Interactions that may be involved in biomolecular condensates.

\subsection{Associative LLPS: coacervate-based synthetic cells}

The other common type of LLPS in aqueous solutions involves the formation of a colloid-rich liquid phase in equilibrium with a diluted phase through associative LLPS (Figure 1). For macromolecules, this condensation can be described in terms of the Flory--Huggins theory, which predicts a first-order transition in cases of sufficiently strong interactions, resulting in the coexistence of two liquid phases (Figure 1D). $[6,16]$

The term coacervation was originally introduced by Bungenberg de Jong in the 1920s specifically to describe the associative phase separation between two oppositely charged natural polyelectrolytes. ${ }^{[17]}$ Since then, two limiting cases of associative phase separation, dominated either by desolvation or by electrostatic interactions, have been identified, although associative LLPS can also be driven by other attractive interactions (as discussed below for biomolecular condensates). Whereas simple coacervation refers to systems containing only one colloidal component undergoing phase separation due to decreased solvation (e.g., through addition of a dehydrating chemical agent or changes in temperature or $\mathrm{pH}$ ), complex coacervation describes a spontaneous phase separation resulting from attractive electrostatic interactions of two oppositely charged molecules or colloids in aqueous solution. Complex coacervates have been produced from a number of colloidal objects, including hundreds of different synthetic or natural polyelectrolytes, proteins, ${ }^{[18]}$ shorter polyions, such as oligopeptides ${ }^{[19]}$ and oligonucleotides, ${ }^{[20]}$ and small molecules, such as mononucleotides ${ }^{[21]}$ and surfactants. ${ }^{[22]}$ 
It has been proposed that the forces driving the phase separation of two oppositely charged long polyelectrolytes originate from entropy increases associated with the release of counter-ions and rearrangements of water molecules during the formation of macro-ion pairs. ${ }^{[23]}$ These pairs further assemble to produce a liquid-like dense microphase. In some cases, the polyelectrolyte pair eventually precipitates to form a solid-like complex, but the parameters influencing the nature of the complex formed (liquid or solid) for a given polyelectrolyte pair remain unclear. ${ }^{[24]}$ Other open questions include the detailed roles of charge distribution and density, long-range electrostatics, hydration effects and contributions of other interactions (hydrogen bonds, cation-- $\pi, \ldots$... Recent studies in this area have sought to understand these factors in greater detail by using computational modelling combined with better-defined polyelectrolyte structures, ${ }^{[25]}$ in terms of, for example, polymer length dispersity, ${ }^{266}$

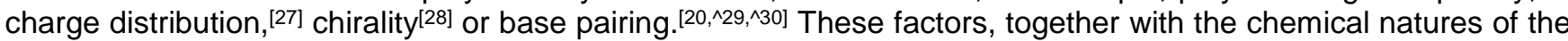
species involved (long polyelectrolytes or small molecules), influence the material properties of the coacervate phase produced, including the rheological and interfacial properties. ${ }^{[31]}$ The ease of coacervate formation and the diversity of assembly blocks have provided applications in areas as diverse as food thickening, ${ }^{\left[{ }^{[3]}\right.}$ cosmetic $^{[33]}$ or pharmaceutical[25b] formulations, protein purification, ${ }^{[34]}$ wastewater treatment ${ }^{[35]}$ or underwater adhesion. ${ }^{[36]}$

In the mid-1920s, Oparin became the first to suggest that liquid-like microdroplets formed by complex coacervation in water could have played a key role in the emergence of the first metabolic cells. ${ }^{[37]}$ These coacervate droplets were shown to undergo fission, to favour chemical enrichment through spontaneous solute sequestration and to support enzyme-mediated reactions. ${ }^{[38]}$ This idea has re-emerged in recent years with pioneering studies by Mann and co-workers, who demonstrated for the first time the formation of complex coacervate droplets from lowmolecular-weight mononucleotides and their use as viable protocell models. ${ }^{[21]}$ Since then, bio-inspired functions have been demonstrated in a variety of other membrane-free droplets based on complex coacervates, including surfactant-based protocells. ${ }^{[39, \wedge 40]}$

\subsection{Bridging the gap with living cells: intracellular biomolecular condensates}

In recent years, it has become widely acknowledged that liquid-liquid phase separation processes play a crucial role to organize contents in eukaryotic cells and bacteria. ${ }^{[4]}$ These intracellular LLPS phenomena produce liquidlike micro-droplets known as "membrane-less organelles" or "biomolecular condensates", among which are the cytoplasmic P granules, the nucleolus and the nuclear Cajal bodies. ${ }^{[35]}$ Their liquid-like behaviour is supported by their ability to flow under shear, fuse and relax to spherical droplets. Although the driving forces of formation of these droplets have not yet been completely elucidated, it has been found that biomolecular condensates often rely on intrinsically disordered proteins containing low complexity sequences of charged residues, ${ }^{[36]}$ and possibly RNA. In contrast to most synthetic LLPS, biomolecular condensates involve many different types of weak and multivalent interactions that affect the materials property of the droplets formed ${ }^{[37]}$ (Figure 1C). A full description of these interactions is very challenging, ${ }^{[24]}$ but quantitative image analysis, together with in vitro reconstitution using well-defined solution compositions may shed light on the physicochemical properties of these systems. ${ }^{[38]}$

Biomolecular condensates show intricate dynamics: ${ }^{[5]}$ their formation and dissolution is rapidly triggered by subtle changes in their concentration or in the solution conditions, and they easily exchange matter with their surroundings. These properties rely on either passive or active processes that confer to the droplets specific material properties, ensure selective partitioning of biomolecules or prevent aging and fusion. ${ }^{[39]}$ Living cells exploit membrane-free compartmentalization for a variety of cellular functions, ${ }^{[40]}$ including spatiotemporal regulation of biochemical reactions, ${ }^{[4,41]}$ contents organization during division, ${ }^{[42]}$ signal amplification, integration, or attenuation via passive noise filtration to only detect and respond to relevant signals. ${ }^{[43]}$ Changes in the materials properties of liquid-like droplets has further been related to diseases, e.g. due to arrested dynamics (gelation) and protein fibril formation. ${ }^{[44]}$ The rich diversity of functional and material properties of biomolecular condensates, together with the fine spatiotemporal control over biochemical reactions they offer in response to subtle changes in their environment, are now inspiring the design of dynamic synthetic cells based on LLPS.

\section{Biomolecule exchange and biochemical activity: "open" vs. "closed" compartments}

\subsection{Biomolecular partitioning and biochemical reactions in membrane-free droplets}

Owing to the lack of membrane on micro-droplets formed by all-aqueous LLPS, solutes added to the continuous phase easily diffuse across the droplet interface, a feature that is characteristic of two phase systems. ${ }^{[45]}$ It is observed that solutes are either sequestered by or excluded from the droplets. ${ }^{[20,46,47]}$ Membrane-free droplets can hence be described as "open" reactors, capable of rapid molecular exchanges with their environment without the need of active transport machineries (Figure 2A). These favourable properties are exploited by living cells to rapidly regulate biochemical reactions, and may have potential implications in the emergence of functional protocells capable of selective binding, chemical reactivity (e.g. catalysis, proto-metabolism) and genetic evolution (e.g. synthesis of oligonucleotides from mononucleotides). ${ }^{[48]}$ Although the driving forces behind selective solute partitioning remain poorly understood, factors such as the charge, hydrophobicity, size, or shape of biomolecular solutes, together with the chemical nature of the LLPS components, have been invoked to account for their uptake or exclusion by aqueous droplets. This differential solute sequestration is quantified experimentally by measuring 
an equilibrium partition coefficient, usually defined as the solute concentration within the droplets divided by the solute concentration in the continuous phase.

Low molecular weight solutes do not usually exhibit high partitioning in neutral polymer ATPS. ${ }^{[7]}$ In contrast, complex coacervate droplets have been found to sequester small solutes, including ions, ${ }^{[49]}$ based on complimentary electrostatic interactions, together with hydrophobic effects associated to the lower dielectric constant of the droplets interior compared to the dilute phase. ${ }^{[18,20,46]}$ Small biomolecules have also been used as scaffold solutes to assemble membrane-free droplets. ${ }^{[20,46,49]}$ In a notable example, Koga et al. demonstrated the formation of coacervate micro-droplets from nucleoside triphosphates (ATP), diphosphates (ADP, FAD, NAD) or monophosphates (AMP) mixed with oppositely charged oligolysine chains, and reported a 20 -fold increase in ATP concentration relative to the dilute continuous phase. ${ }^{[20]}$ Oligo- and polynucleotides also partition unevenly in LLPSbased droplets. In PEG/dextran ATPS, single-stranded RNA[50] and double-stranded DNA ${ }^{[51]}$ preferentially accumulate in the dextran phase, with increased partition coefficients reported for higher polynucleotides lengths. ${ }^{[50,51]}$ The polynucleotide exchange rate between the droplets and their environment appears to be highly system-dependent. For instance, rapid exchange of RNA oligomers was reported in PEG/dextran ATPS and polylysine/ATP coacervate droplets; ${ }^{[52]}$ yet, very little exchange of single-stranded DNA oligomers was observed for up to 48 hours in a binary poly(diallyldimethylammonium bromide) (PDDA)/ATP coacervate droplets population produced by microfluidics. ${ }^{[53]}$ The secondary structure ${ }^{[54]}$ and length[55] of RNA, as well as the length and single- vs. double-stranded nature of DNA ${ }^{[56]}$ were reported to affect their partitioning, which was attributed, in the latter case, to a characteristic mesh size of the coacervate matrix, resulting in the exclusion of longer and stiffer doublestranded DNA chains. ${ }^{[56]}$ In contrast, RNA length had no influence on its partitioning in poly(allylamine)/ATP coacervate droplets, where another sequestration mechanism based on the exchange of ATP molecules for RNA macromolecules within the droplets was postulated. ${ }^{[49]}$ While dissociation of nucleic acid duplexes was reported in protein-based droplets, ${ }^{[56]}$ the co-sequestration of complementary oligonucleotides in surfactant based protocells was found to promote base pairing. ${ }^{[34]}$

The sequestration of proteins in membrane-free droplets has also been extensively investigated. In the case of PEG/dextran ATPS, native proteins tend to partition preferentially in the more hydrophilic dextran phase, while denatured proteins have been shown to exhibit a stronger affinity for the more hydrophobic PEG phase. ${ }^{7,57]}$ The structure of proteins was also found to affect their partitioning in complex PDDA/PAA coacervate droplets: native proteins were sequestered in the droplets, but were released in the dilute phase upon urea-mediated unfolding, a property that was used to achieve coacervate-assisted protein refolding. ${ }^{[58]}$ In comparison, the sequestration of BSA within PAA/PAH coacervate droplets provided protection against protein denaturation when exposed to nonphysiological $\mathrm{pH}$, high temperatures, or urea. ${ }^{[59]}$ Other factors including the ionic strength, ${ }^{[60]}$ the order of addition of the coacervate components vs. protein ${ }^{[61]}$ or the polyelectrolyte ratio[60] were further shown to affect protein sequestration. Rather than being added to pre-formed coacervate droplets, proteins can also act as scaffold components in biomolecular condensates ${ }^{[62]}$ as well as in synthetic systems. ${ }^{[17,63]}$ Chemical modifications have recently been used to increase the propensity of proteins to undergo liquid-liquid phase separation, e.g. via protein supercharging ${ }^{[64]}$ or via covalent attachment of ionic polypeptide tags. ${ }^{[65]}$

The sequestered biomolecules remain highly dynamic within the liquid-like droplets, although they can experience sub-diffusive transport.[66] How their localized up-concentration within a crowded phase affect their biochemical reactions is an area of current study. Co-localization of partner reactants (e.g. enzymes and substrates) may accelerate biochemical reactions. ${ }^{[67]}$ For instance, the accumulation of hammerhead ribozymes within the dextran-rich phase of a PEG/dextran ATPS was reported to enhance RNA catalysis rates, due to a combination of localized RNA up-concentration and crowding effects. ${ }^{[50]} A$ similar reaction was recently reported in carboxymethyl dextran/poly-L-lysine micro-droplets, indicating that complex coacervation could also support RNA catalysis, although rates 13 - to 60-fold slower than in buffer were observed[55] (Figure 2B). In another notable example, enhanced ribozyme cleavage yields were reported in complex coacervates formed by PDDA and oligoaspartic acid, which was attributed to the local up-concentration of RNA within the droplets. ${ }^{[68]}$ Significantly, by reconciling membrane-free compartmentalization and RNA catalysis, these studies provide possible routes towards the emergence of proctocells capable of RNA-based catalytic activity and genetic information storage on the primordial Earth, in line with the RNA world hypothesis. ${ }^{[69]}$ In other studies, phase separation of short DNA or RNA oligomers from a PEG-rich solution was found to produce droplets with liquid crystal ordering, which enhanced the chemical end-to-end ligation efficiency of the short strands into longer oligonucleotides. ${ }^{\text {[0] }}$ These studies demonstrate that supramolecular ordering is sufficient to favour oligonucleotide templating and enhance nonenzymatic oligonucleotide polymerization. More complex, multi-step reactions were also realized in ATPS and coacervate droplets, including cell-free transcription-translation ${ }^{[71]}$ and concerted cascade enzyme reactions, ${ }^{[72}$ with reported enhanced reaction rates or yields.

Conversely, biochemical reactions may also be prevented or retarded in LLPS-based droplets. The different local environment experienced by biomolecules (e.g. in terms of viscosity, dielectric constant, $\mathrm{pH}$...) may affect their activity. In addition, the differential sequestration of enzymes, substrates, intermediates and products within the droplets may result in decreased reactivity (e.g. if the droplets sequester an enzyme but exclude its substrate). Experimental and theoretical studies in ATPS showed only poor reaction rate enhancement for a pair of enzymes involved in a concerted cascade reaction due to the low partitioning of enzymes in the droplets. ${ }^{[73]}$ The phenomenon known as substrate inhibition has also been reported as a plausible cause of reduced enzyme reactivity: in a study, dextranase was shown to be inactivated when sequestered in dextran-rich droplets due to the high local 
concentration of substrate dextran macromolecules ${ }^{[74]}$ By compartmentalizing dextranase in a coacervate subcompartment within the dextran droplet, substrate inhibition was mitigated and dextranase-mediated interfacial dextran hydrolysis was restored. ${ }^{[74]}$

Overall, these studies indicate that the extent of biomolecules partitioning into one or the other phases produced by LLPS may strongly affect the rate of biochemical reactions. Rationalizing the connections between differential partitioning, up-concentration, crowding, chemical composition, protein conformation etc. and the structure, conformational stability and activity of enzymes in membrane-free droplets is an area of increasing interest that will help designing robust and versatile bioactive droplets capable of more advanced functions (e.g. feedback, control, logical operations etc.).

A) "Open" reactor

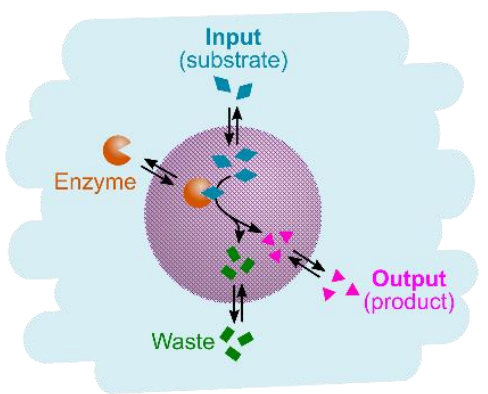

B)
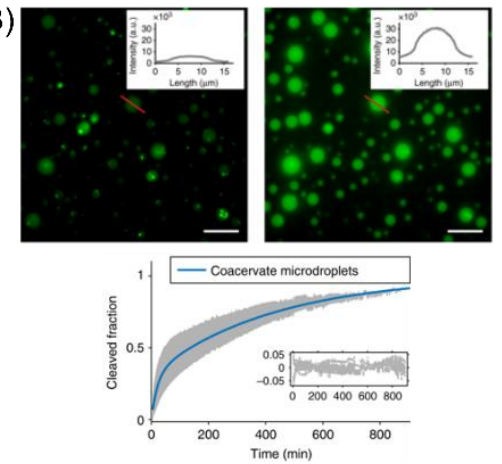

C)

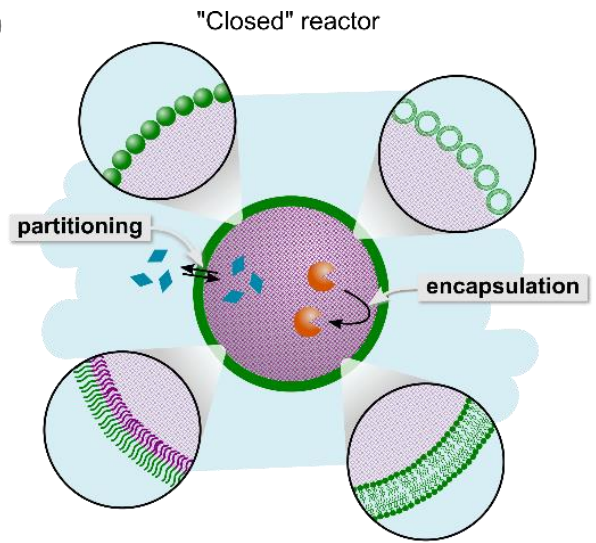

D)

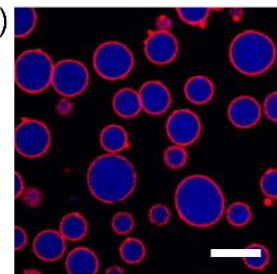

F)

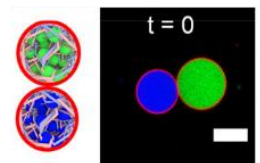

E)

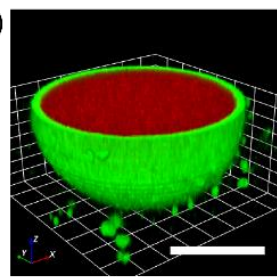

G)

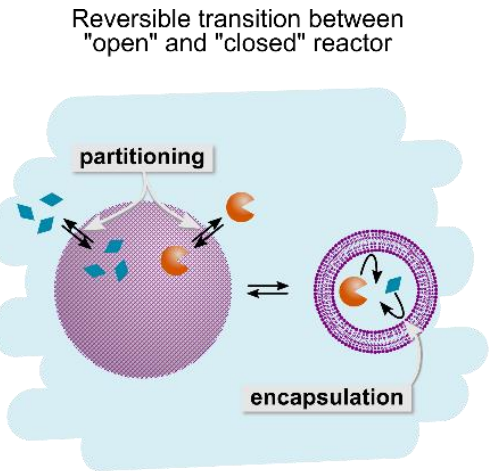

H)

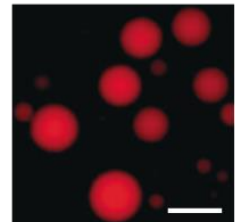

I)

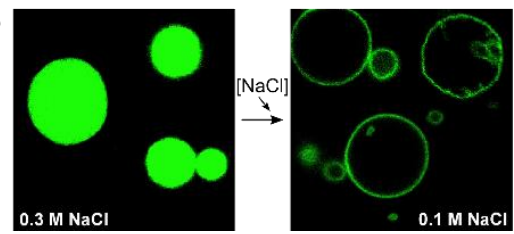

Figure 2. A) Schematic representation of LLPS-based droplets as "open" reactors, capable of matter exchange with the environment. B) RNA catalysis in carboxymethyl dextran/poly-L-lysine coacervate micro-droplets, showing localized increase of fluorescence in the droplets due to cleavage of an RNA-based substrate (scale bars $=20 \mu \mathrm{m}$ ). Adapted from ref. 55 published under a CC BY 4.0 license. C) Schematic representation of LLPS-based droplets as "closed" reactors, where a semi-permeable membrane is assembled at the surface of LLPS-based droplets. D-F) Examples of membrane-stabilized LLPS-based droplets: dextran-in-PEG droplets decorated by small unilamellar vesicles (D), scale bar $=10 \mu \mathrm{m}$ ) or amine-modified polystyrene nanoparticles $(\mathrm{E})$, scale bar $=5 \mu \mathrm{m})$, and amylose-based complex coacervates stabilized by copolymers $(F)$, scale bars $=10 \mu \mathrm{m}$ ), showing no exchange of biomacromolecular content. Image in D) adapted with permission from ref. 79 . Copyright 2015, American Chemical Society. Image in E) Reproduced with permission from ref. 77. Copyright Wiley-VCH Verlag GmbH \& Co. $\mathrm{KGaA}$, Weinheim. Image in F) adapted from ref. 89 (any further permissions related to this image should be directed to the American Chemical Society). G) Schematic representation of reversible transformation between membrane-free LLPS-based droplets ("open" reactors) and membrane-bound compartments ("closed" reactors). $\mathrm{H}$ ) Example of pH-mediated reversible transition between fatty acid micelle coacervate droplets (left, scale bar $=50 \mu \mathrm{m}$ ) and fatty acid vesicles (right, scale bar $=5 \mu \mathrm{m}$. Inset shows the encapsulation of YFP (pre-sequestered in the droplets) within the vesicles (scale bar $=1 \mu \mathrm{m}$ ). Reproduced and adapted with permission from ref. 33. Copyright Wiley-VCH Verlag GmbH \& Co. KGaA, Weinheim. I) Confocal fluorescence images of the transformation of coacervates (left) into polymersomes (right) by dialysis. Reprinted from ref. 91 with permission from Elsevier. Copyright 2017 Elsevier Ltd.

\subsection{Controlled matter exchanges via interfacial membrane assembly}

Although the lack of membrane on droplets formed by LLPS confers them the ability to act as open reactors by facilitating matter exchange with the environment, it also favours low confinement times due to passive solute diffusion in and out of the droplets, together with spontaneous droplet fusion (or coalescence). The design of droplets capable of resisting fusion and molecular exchange while retaining selective solute partitioning has emerged as a promising strategy to produce discrete synthetic cells with prolonged lifetime and controllable matter exchanges ("closed" or "semi-closed" compartments, Figure 2C).

An approach directly inspired from conventional emulsion stabilisation procedures to prevent coalescence of allaqueous droplets relies on the interfacial adsorption of surface-active molecules or particles, which has led several groups to describe such droplets as water-in-water (W/W) emulsions. ${ }^{[75]}$ Albeit conceptually trivial, the stabilization of W/W emulsions by surface-active species is technically challenging due to the very low interfacial tensions involved. Spherical colloids with a diameter typically larger than $100 \mathrm{~nm}$, including nanoparticles, ${ }^{[76,77]}$ lipid vesicles, ${ }^{[78,79]}$ and protein clusters, ${ }^{[80]}$ as well as high aspect-ratio colloids, such as clays, ${ }^{[81]}$ nanorods ${ }^{[82]}$ or protein fibrils, ${ }^{[83]}$ adsorb more effectively to interfaces with low surface tension, ${ }^{[83,84]}$ and have therefore been used to stabilize PEG/dextran aqueous two-phase systems (Figure 2D,E), as well as complex coacervate droplets. ${ }^{[85,86]}$ 
Membranes obtained after the interfacial assembly of such large colloidal objects are usually highly permeable to molecular and macromolecular species due to the large size of the pores created. This non-selective permeability is advantageous to form discrete bioreactors (stabilized against coalescence) that retain the capacity to spontaneously sequester and accumulate biomolecules from the continuous phase (due to their equilibrium partitioning within the droplets). ${ }^{[78,79]}$ For instance, liposome-stabilized dextran droplets were shown to spontaneously uptake ribozymes from the continuous solution due to the preferential partitioning of the polynucleotides within the droplets, resulting in localized cleavage of short oligonucleotide substrates partitioned in the dextran-rich droplets. ${ }^{[78]}$ In another study, polystyrene nanoparticles were successfully adsorbed and crosslinked at the surface of PEG-suspended dextran-rich droplets to produce stable all-in-water colloidosomes. ${ }^{\text {[77] }}$ Significantly, the so-formed capsules were able to spontaneously uptake fluorescently labelled dextran macromolecules from the continuous solution due to their permeation through the large membrane pores and their preferential partitioning within the droplets.

However, matter exchanges in living cells are usually more finely regulated, and exploit encapsulation within membrane-bound organelles to limit passive solute exchanges. Building upon these ideas, the construction of micro-compartments combining a crowded interior and a closely packed, imper- or semi-permeable membrane holds great promise to achieve local chemical enrichment, prolonged molecular encapsulation and regulation of matter exchanges. In a notable example, Tang et al. successfully produced PDDA/ATP complex coacervate microdroplets coated with a fatty acid membrane by direct addition of oleic acid molecules to the droplets suspension. ${ }^{[87]}$ Significantly, the lipid membrane limited droplet coalescence and provided differential permeability to dyes depending on their charge and molecular weight. Macromolecular design has also been used to synthesize triblock co-polymers able to stabilize droplets formed by LLPS in water, including PEG/dextran aqueous two-phase systems ${ }^{[88]}$ and, more recently, amylose-based coacervate droplets ${ }^{[89]}$ (Figure 2F). These structures provide new routes to hybrid protocellular compartments that combine aspects of membrane-mediated encapsulation and localized chemical enrichment.

\subsection{Droplet-to-vesicle transformations: reversible switching between "open" and "closed" compartments}

An exciting alternative strategy to dynamically control matter exchanges has recently emerged based on systems able to transform between membrane-free droplets and membrane-bound vesicles (Figure 2G). The first study to demonstrate such a droplet-to-vesicle transition involved the spontaneous transformation of PDDA/ATP coacervate micro-droplets into capsules by addition of polyoxometalates (POM). ${ }^{[90]}$ In this work, electrostatic binding of anionic phosphotungstate clusters to the surface of the positively charged droplets induced the formation of a water-rich vacuole within the coacervate matrix due to the osmotic pressure established across the semi-permeable membrane. This resulted in the production of a stable vesicular-like structure with a well-defined molecular weight cut-off, but the transformation of coacervate droplets into capsules was not reversible.

Following this seminal work, Douliez and co-corkers were the first to demonstrate a reversible coacervate-tovesicle transition in myristic acid micelles micro-droplets, activated by changes in $\mathrm{pH}^{[33]}$ (Figure $\left.2 \mathrm{H}\right)$. At high $\mathrm{pH}$ (above the fatty acids pKa), myristic acid molecules self-assembled into elongated micelles that phase separated to produce micelle-rich membrane-free droplets in the presence of excess guanidinium counter-ions. Changing the molecular packing parameter (by decreasing the $\mathrm{pH}$ below the fatty acids $\mathrm{pKa}$ ) resulted in the stabilization of fatty acid bilayers and the associated formation of membrane-bound fatty acid vesicles. Very excitingly, a substantial fraction of biomolecules accumulated in the coacervate micro-droplets was spontaneously transferred in the lumen of the vesicles after the $\mathrm{pH}$-induced transition. ${ }^{[33]}$ This was the first report to demonstrate reversible transformation between membrane-free and membrane-bound compartments, together with spontaneous solute encapsulation. Although preliminary in design, this dual compartmentalized organization could have strong implications in the origin of the first metabolic protocells. Significantly, reversible transition between membrane-free and membranebound structures allows cycling between open and closed reactors, providing new opportunities to sustain metabolic reactions, e.g. by spontaneous nutrient uptake and waste disposal in the membrane-free state (due to equilibrium partitioning) and long-term biomolecular segregation and localized reactions (proto-metabolism, DNA replication, etc.) in the membrane-bound state.

Owing to their net negative charge, fatty acid-based coacervate droplets did not sequester polynucleotides. To circumvent this limitation and further develop this system into viable protocell models, Douliez and co-workers successfully produced catanionic surfactant coacervate droplets capable of both $\mathrm{pH}$-mediated reversible coacervate-to-vesicle transition and DNA sequestration due to the presence of positively charged surfactants. ${ }^{34}$ While much work remains, this study demonstrates that reversible coacervate-to-vesicle transformations could be used to promote spontaneous transfer of DNA from membrane-free to membrane-bound compartments, and ultimately sustain genetic information replication via substrate uptake and waste disposal. Future works will need to extend this reversible coacervate-to-vesicle transition to other systems, in particular to produce more stable vesicles (compared to leaky fatty acid membranes). It is here worth mentioning that a recent study reported the transformation of polymer-based coacervate droplets into polymersomes by changes in the ionic strength ${ }^{[91]}$ (Figure 21). Another notable study demonstrated that the assembly of vesicles from a model co-polymer via solvent switching was preceded by the transient formation of polymer-rich liquid droplets that favoured the nucleation of polymer micelles and their evolution into bilayers. ${ }^{\left[{ }^{[2]}\right]}$ These studies therefore suggest that coacervate-to-vesicle transitions may be a general phenomenon that could be extended to other building units. 


\section{Dynamic compartmentalization via reversible phase separation}

Many intracellular biomolecular condensates are highly dynamic at the micron-scale, and can readily be formed and dissolved in response to environmental cues. ${ }^{[5]}$ This dynamic organization confers cells with the ability to regulate the spatial distribution of functional components and orchestrate biochemical reactions. Reproducing such a responsive phase separation behaviour in synthetic systems holds great promise for the spatiotemporal regulation of (bio)chemical reactions in synthetic cells (Figure $3 \mathrm{~A}$ ).

A)

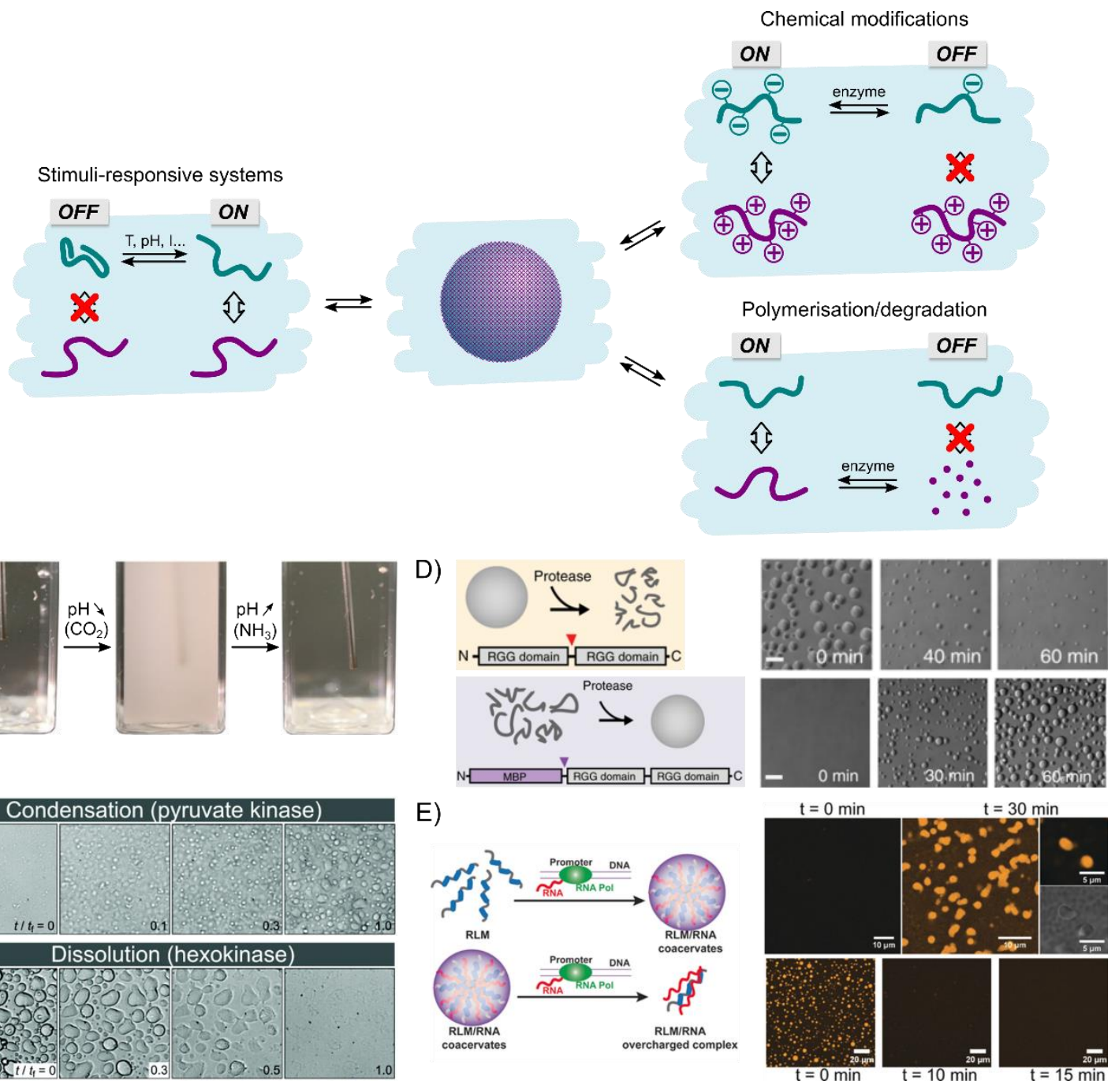

Figure 3. A) Schematic representation of different scenario of responsive LLPS. B) Pictures showing pH-driven reversible assembly of oligolysine/ATP coacervate droplets. Adapted by permission from Springer Nature, Nature Chemistry, ref. 20, copyright 2011. C) Optical images showing condensation and dissolution of ATP/poly-L-lysine coacervate droplets in response to enzyme activities. Adapted from ref. 110 published by The Royal Society of Chemistry under a CC BY-NC 3.0 license. D) Control of protein-based droplet dissolution and condensation in response to proteolytic activity (scale bars $=10 \mu \mathrm{m}$ ). Adapted from ref. 115 published under a CC BY 4.0 license. E) Cell-free DNA translation drives liquid-liquid phase separation in the presence of oligopeptides, followed by droplets dissolution due to the formation of overcharged complexes. Reprinted with permission from ref. 117. Copyright 2017, Wiley-VCH Verlag GmbH \& Co. KGaA, Weinheim.

\subsection{Stimuli-responsive phase separation}

Due to the weak interactions at play between phase-separating species, changes in the solution conditions, such as the ionic strength, $\mathrm{pH}$ or temperature, may affect phase separation without altering the chemical nature or concentration of the species involved. The addition of inorganic salts has commonly been used to disassemble droplets formed by complex coacervation between oppositely charged polyelectrolytes, due to the decreased

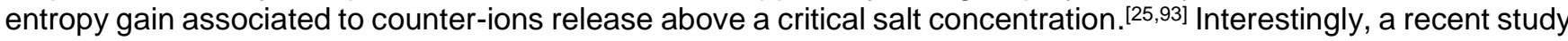
reported a switch-like behaviour in RNA-based droplets in response to divalent cations, where heterotypic or homotypic droplets were formed depending in the concentration of magnesium ions. ${ }^{[94]}$ However, the addition of salt is hardly reversible. The use of stimuli-responsive species able to phase separate by changing the intensive solution parameters, such as the $\mathrm{pH}$ or temperature, offers a clear advantage to achieve reversible control over phase separation. Several studies have reported the influence of $\mathrm{pH}$ on complex coacervation formed from weak 
polyelectrolyte due to changes in their protonation state. ${ }^{[95]}$ In a notable example, opposite $\mathrm{pH}$ changes have been used to favour the reversible assembly of coacervate micro-droplets formed by poly-L-lysine (PLys) and ATP due to alterations in the protonation state of PLys ${ }^{[20]}$ (Figure 3B).

Temperature-sensitive biomolecular condensation has also recently been demonstrated in living cells, with reported examples of either phase separation or favoured mixing above a critical temperature threshold or during heat stress. ${ }^{[96-98]}$ For example, liquid-like droplets formed by Ddx4-based protein constructs were shown to form below a threshold temperature and to reversibly dissolve upon heating, ${ }^{[97]}$ while the spindle-associated BuGZ protein was shown to undergo phase separation upon heating. ${ }^{[98]}$ In comparison, the design of thermo-responsive polymers capable of undergoing condensation upon heating (lower critical solution temperature (LCST)-type behaviour) or cooling (upper critical solution temperature (UCST)-type behaviour) has been widely documented in materials science. ${ }^{[99]} \mathrm{A}$ wide class of naturally occurring polymers capable of thermally driven simple coacervation in water includes elastomeric proteins, such as elastins or resilins. ${ }^{[100]}$ Chilkoti and co-workers have demonstrated that the thermo-responsive behaviour of such proteins could be encoded in the sequence of amino acids, ${ }^{[101]}$ providing a simple approach to program the phase separation behaviour via the rational design of the protein sequence. ${ }^{[102]}$ In a recent study, thermally driven simple coacervation of polynucleotides was demonstrated based on purine containing single-stranded DNA chains that were found to exhibit a LCST-type behaviour. ${ }^{[103]}$ Thermally reversible complex coacervation of short polyamines and low complexity RNA was also demonstrated due to temperature-mediated conformational changes of the latter that altered its interaction with the amines. ${ }^{[85]}$ In the case of aqueous two-phase systems, increased or decreased miscibility of PEG and dextran were reported depending on the temperature. Keating and co-workers used these observations to encapsulate a single-phased $\mathrm{PEG} /$ dextran solution in giant lipid vesicles at high temperature, then trigger phase separation by lowering the temperature. ${ }^{[14]}$ Interestingly, fluorescently labelled proteins that were initially homogenously distributed throughout the vesicles partitioned preferentially in the dextran phase after phase separation, providing an example of dynamic control of protein localization via liquid-liquid phase separation.

Motivated by the tight spatiotemporal regulation of biomolecular condensates in living cells, light-responsive building blocks are now being developed to achieve light-induced LLPS. In a notable example, Brangwynne and co-workers designed protein constructs capable of self-association and subsequent liquid-liquid phase separation under light stimuli, resulting in spatiotemporal control of biomolecular condensation in living cells. ${ }^{[104]}$ A lightresponsive aqueous two-phase system based on PEG and spiropyran-functionalized dextran macromolecules was also reported. ${ }^{[105]}$ These studies open new perspectives for the spatiotemporal control of LLPS.

\subsection{Enzyme-responsive phase separation: coupling structure and processes}

Biochemical modifications of LLPS-prone species. A central hallmark of living cells is that structures and processes are closely connected: notably, biomolecular condensates are finely regulated in space and time via biochemical reactions, such as posttranslational protein or RNA modifications. For instance, phosphorylation reactions were shown to be tightly coupled to RNA granule formation and dissolution, ${ }^{106]}$ and to alter phase separation of a lowcomplexity domain protein. ${ }^{[107]} \mathrm{A}$ study further demonstrated the prominent role of kinase activity on biomolecular condensation during cell division. ${ }^{[108]}$ Arginine methylation was also shown to promote the dissolution of Ddx4based droplets in vitro. ${ }^{[97]}$

Inspired by these biological regulation processes, enzyme-mediated chemical modifications of phase-separating species are now emerging as promising alternatives to dynamically control and program liquid-liquid phase separation in synthetic systems. In a study by Keating and co-workers, a kinase/phosphatase enzymes pair was used to alter the net positive charge of short oligopeptides via phosphorylation/dephosphorylation reactions, therefore regulating their complex coacervation capacity with negatively charged RNA macromolecules. ${ }^{[109]}$ This process was shown to be reversible by modulating the enzyme activities; however, the two opposite enzyme reactions were not mutually compatible, and addition or removal of specific enzyme co-factors was required to achieve reversibility. In another example, Spruijt and co-workers demonstrated formation and dissolution of polylysine/ATP coacervate micro-droplets under the same solution conditions by using another kinase/phosphatase pair to reversibly convert ATP into ADP[110] (Figure 3C). Excitingly, temporal programming of droplet dissolution and condensation was also demonstrated in this work by modulating the rates of phosphorylation or dephosphorylation reactions. Another recent work reported the use of cyclodextrin and amylase to enzymatically regulate in space and time the formation and dissolution of complex coacervate droplets formed from two oppositely charged small molecules. ${ }^{[111]}$ These pioneering studies pave the way to the design of self-regulated droplets which formation and dissolution is controlled by enzyme reactions. Future directions will need to explore more complex enzyme networks, e.g. to introduce self-sustained phase separation or feedback control, and couple the formation/dissolution of droplets to biomolecule uptake/release.

Biochemical alteration of the concentration of LLPS-prone species. Intracellular biomolecular condensation is also strongly dependent on the concentration of phase separating species, with several observations pointing to the existence of a critical threshold concentration below which intracellular phase separation no longer occurs. ${ }^{[112]}$ This is similar to synthetic systems, for which phase diagrams clearly established that phase separation occurs above threshold concentrations of phase-separating species. Altering the concentration of phase separating species hence provides another mechanism to control liquid-liquid phase separation. Osmotic pressure differences across a semi-permeable membrane have been exploited to induce changes in the local concentrations of 
encapsulated phase-separating species and therefore induce phase separation. For instance, phase separation of bacterial cell lysate encapsulated in water-in-oil droplets was achieved via osmotically driven up-concentration of the lysate, which resulted in increased transcription rates. ${ }^{[71]}$ Similarly, multistep osmostic shocks applied to lipid vesicles containing cell lysate and PEG were shown to induce lysate phase separation due to liposome shrinkage, resulting in localized protein expression. ${ }^{[113]}$

In comparison, the concentration of species forming biomolecular condensates is regulated via DNA transcription and translation (to produce RNA and proteins), together with biochemical degradation. Building upon these ideas, approaches based on enzyme-mediated hydrolysis or cell-free gene transcription tools to control synthetic LLPS are now being investigated. For instance, dextranase has been used to hydrolyse dextran macromolecules into smaller oligosaccharides and hence trigger the dissolution of dextran-rich droplets suspended in a PEG-rich phase ${ }^{[74]}$ or embedded within a hydrogel, ${ }^{[114]}$ resulting in the gradual release of sequestered proteins. A significant advance for the triggered assembly and dissolution of liquid-like micro-droplets was recently achieved by the rational design of intrinsically disordered protein (IDP) constructs containing engineered protease cut sites and protein interaction motifs ${ }^{[115]}$ (Figure 3D). In this work, the authors achieved controllable phase separation in response to protease-mediated targeted hydrolysis of the IDP constructs, resulting in programmable recruitment and release of cargo proteins. In another study by Huck and co-workers, out-of-equilibrium enzymatic networks producing oscillations in protease concentration were coupled to the formation and dissolution of coacervate droplets due to protease-mediated hydrolysis of one of the coacervate components. ${ }^{[116]}$ The biosynthesis of phase separating species has also been exploited to trigger phase separation in vitro. The first example to demonstrate such a mechanism was reported by Deniz and co-workers who used a cell-free DNA transcription toolkit to produce RNA macromolecules that phase-separated in the presence of positively charged oligopeptides ${ }^{[117]}$ (Figure 3E). The authors further demonstrated that the droplets formed could be dissolved by excess synthesis of RNA due to charge inversion and associated long-range electrostatic repulsion, a phenomenon known as re-entrant phase transition. ${ }^{[118]}$

Taken together, these studies highlight new opportunities for the reversible control of liquid-liquid phase separation based on biochemically-regulated changes in the concentration or chemical nature of phase-separating species. They also open new perspectives for the development of biochemical reaction networks allowing selfregulation of synthetic cells.

\section{Droplet growth and division}

The design of model micro-compartments capable of growth and division holds great promise to understand the minimal requirements to the self-reproduction of protocell on the early Earth, and represents a crucial step for the fabrication of synthetic cells capable of duplication, proliferation, and, ultimately, evolution. ${ }^{[119,120]}$ Growth and division have historically been investigated in membrane-bound model systems, including fatty acid[121,122] and lipid[119] vesicles. However, all-aqueous droplets produced by liquid-liquid phase separation provide promising alternative models for the construction of protocells capable of dynamic growth and division.

\subsection{Droplet growth}

Cell growth is a complex dynamic process that involves extensive structural rearrangements, membrane remodelling and duplication of contents to produce two identical second-generation cells. In contrast, growth of allaqueous droplets is achieved via simple mechanisms, ${ }^{[39,120,123]}$ including material uptake from a supersaturated solution of phase-separating species; Brownian motion-induced fusion (or coalescence); and diffusion-limited Ostwald ripening, where phase-separating species are transferred through the continuous phase from smaller droplets to larger ones due to differences in their surface energy (Figure 4A). While Ostwald ripening has been observed in living cells, ${ }^{[120]}$ droplet growth in synthetic LLPS is usually associated to coalescence events. The rate of coalescence can sometimes be modulated, for instance by tuning the charge ratio between components in the case of complex coacervates, but the final average droplet size is usually poorly controlled, and macroscopic phase separation is eventually observed. Droplet-based microfluidics has been used to control droplet growth and produce uniformly sized coacervate droplets ${ }^{[34]}$ or aqueous two-phase systems, ${ }^{[77]}$ but the confinement of the droplets in a water-in-oil emulsion hampers chemical coupling between droplets. An alternative approach was recently developed by Tian et al. who exploited non-contact acoustic forces to promote coacervate droplet growth at specific locations by directed coalescence ${ }^{[124,125]}$ (Figure 4B). Their strategy took advantage of orthogonal acoustic standing waves to pattern coacervate droplets in a two-dimensional array via in situ coalescence of primary droplets into larger, uniformly sized droplets at the acoustic pressure nodes. This notable example demonstrates that directed droplet coalescence is a viable mechanism to induce controlled droplet growth.

In comparison, cell growth is a self-sustained and self-regulated process, ${ }^{[126]}$ a critical feature that is lacking in fusion-based droplets growth. While much work remains, approaches based on the self-production of the own droplets components, e.g. via localized (bio)chemical reactions, such as the transcription of DNA into phaseseparating RNA, ${ }^{[117]}$ may provide viable mechanisms to the autonomous growth of all-aqueous droplets. The young field of systems chemistry may also inspire the construction of self-synthesising droplets, e.g. based on molecular self-replication, with potential implications for the emergence of autonomous protocell duplication on the early Earth. ${ }^{[127,128]}$ 
A)

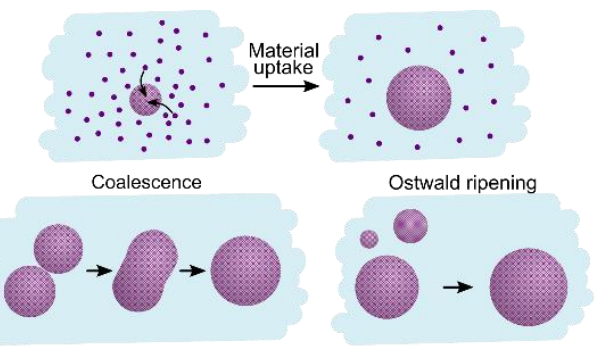

B)

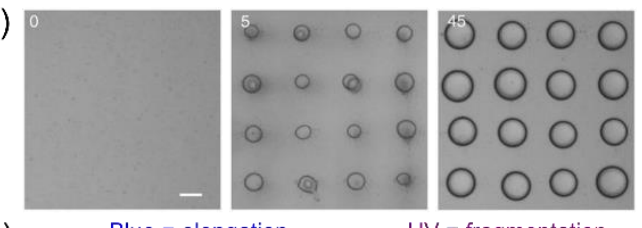

C)

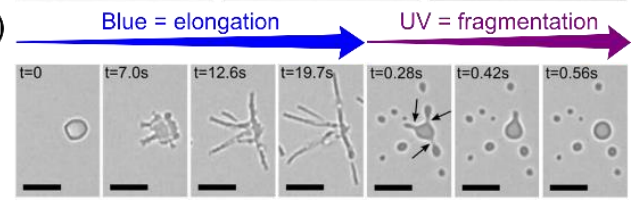

D)

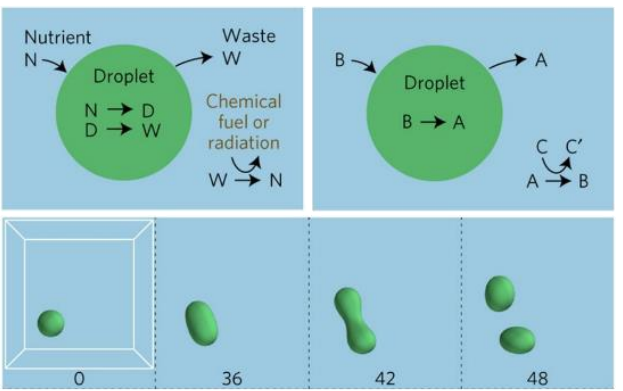

E)

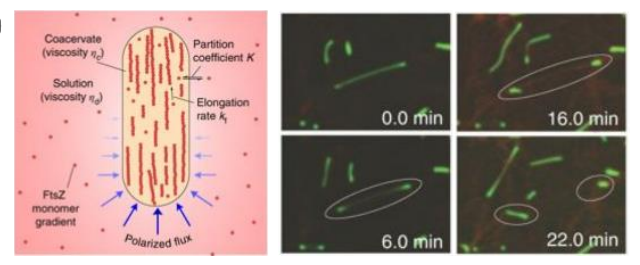

Figure 4. A) Schematic view of processed leading to droplet growth. B) Directed in situ growth of small PDDA/ATP coacervate droplets at the acoustic pressure nodes of orthogonal acoustic standing waves. Scale bar $=50 \mu \mathrm{m}$. Adapted from ref. 124 published under a CC BY 4.0 license. C) Light-driven elongation and self-division of poly(sodium acrylate)/azobenzene microparticles. Adapted from ref. 137 published under a CC BY 4.0 license. D) Chemically active droplets undergo growth and division. Adapted by permission from Springer Nature, Nature Physics ref. 126, copyright 2016. E) Coacervate droplets sequestering FtsZ proteins undergo elongation and fission. Adapted by permission from Springer Nature, Nature Nanotechnology ref. 146, copyright 2018.

\subsection{Spontaneous vs. active droplet fission}

Spontaneous physicochemical processes in LLPS can be exploited to induce division of synthetic cells. For instance, budding and division of dextran-rich micro-droplets suspended in an aqueous PEG solution were reported in the presence of networks of polymerized lysozyme nanofibrils that spontaneously segregated and contracted within the dextran droplets to minimize their interfacial energy. ${ }^{[129]}$ Aqueous two-phase systems enclosed within giant unilamellar vesicles were also found to induce various membrane deformations in response to osmotic perturbations, including wetting-mediated changes in the membrane curvature, budding, and spontaneous formation of membrane nanotubes. ${ }^{[15,130-132]}$ Significantly, complete division was reported for giant unilamellar vesicles encapsulating PEG/dextran ATPS, resulting in the formation of two second-generation vesicles enclosing either a PEG-rich or a dextran-rich phase. ${ }^{[130]}$ Beyond demonstrating that passive processes based on purely physicochemical phenomena may drive asymmetric division in ATPS-enclosing lipid vesicles, this work, together with experimental and theoretical studies on wetting of lipid membranes by ATPS, ${ }^{[131-133]}$ raise questions about the interactions between chemically rich aqueous phases and membranes. These questions are particularly relevant in living cells: for instance, two-dimensional phase separation has been reported at cell membranes, ${ }^{[134]}$ membranelacking compartments have been observed beneath postsynaptic membranes, ${ }^{[135]}$ and liquid-like condensates have been found to exert forces on lipid bilayers resulting in membrane invaginations during endocytosis. ${ }^{[136]}$ Although studies in synthetic systems have for now focussed on ATPS interacting with membranes, it is expected that future studies will also explore the interfacial interactions between complex coacervates and lipid membranes in vitro. 
Division in living cells relies on active, energy-fuelled processes that contrast with the passive fission events discussed above in LLPS-based synthetic cells. The realization of active droplet division is an area of current study. For example, dynamic shaping and self-division of droplets was achieved in response to light energy inputs ${ }^{[137]}$ (Figure 4C): the electrostatic assembly between an azobenzene cation and poly(sodium acrylate) produced soft micro-particles that transformed into liquid-like droplets under UV light due to trans-to-cis azobenzene isomerisation. In comparison, blue light-mediated stochastic trans-cis isomerisations fuelled the elongation of filaments. The latter could then be divided into smaller droplets under UV light due to the associated soft solid-toliquid transition. This energy-fuelled process could be repeated several times to produce multiple light-activated droplet self-divisions. ${ }^{[137]}$

Theoretical studies have further demonstrated that chemically active droplets maintained away from thermodynamic equilibrium undergo shape instabilities resulting in spontaneous droplet division, ${ }^{[126,138]}$ and that cycles of growth and division are achieved in continuously fuelled reactive droplets ${ }^{[126]}$ (Figure 4D). These results could have strong implications in the emergence of division in early metabolic protocells, even before the appearance of membrane-enclosed compartments. Experimental models based on water-in-oil or oil-in-water micro-droplets have been used to demonstrate chemically fuelled self-division due to changes in the droplets surface tension or increase in their surface area via chemical production, resulting in shape instabilities. ${ }^{[128,139]}$ Future experiments will need to demonstrate such chemically active division processes in all-aqueous droplets (ATPS or coacervates) by exploiting localized chemical reactions.

\subsection{Towards droplet division via cytoskeleton reconstitution}

The cellular division machinery consists of a complex ensemble of membrane-binding and cytoskeletal proteins that dynamically re-model the proteolipid bilayer. Remarkably, the cytoskeleton is a dynamic network of proteins filaments (e.g. tubulin and actin in eukaryotic cells, and FtsZ in bacteria) which rearrangements are fuelled by energy-consuming processes (e.g. phosphorylation/dephosphorylation reactions-mediated filament polymerisation/depolymerisation). To shed light on the mechanisms involved in cellular division, the bottom-up reconstitution of cytoskeleton inside synthetic vesicles is under current investigation. ${ }^{[140]}$ A complimentary line of research focuses on cytoskeletal filaments assembly in droplets formed by LLPS to unveil alternative mechanisms involved in cellular division.

Partitioning of the bacterial FtsZ division protein and its filaments were investigated in aqueous two-phase systems, and revealed complex and uneven partition patterns depending on the polymers used. ${ }^{[141]}$ In a recent example, FtsZ proteins were shown to undergo associative LLPS when mixed with the DNA binding protein SmIA in crowding conditions, producing droplets capable of reversibly evolution towards fibres in the presence of GTP. [142] Spontaneous sequestration of actin filaments in PEG-suspended dextran-rich micro-droplets was also reported, and the accumulation of bundled filaments at the droplet surface was shown to induce droplet deformations. ${ }^{[51]}$

Cytoskeleton reconstitution has also been investigated in droplets formed by associative phase separation. For instance, complex polypeptide-based coacervates were shown to spontaneously sequester actin, resulting in an enhanced actin filament assembly rate due to the localized enrichment in cytoskeletal proteins. ${ }^{[143]}$ Based on the low energy cost for interfacial area increase associated to the low surface tensions of all-aqueous droplets, a few studies started to investigate how the local accumulation of polymerizable proteins could produce droplet deformations and, ultimately, division. For instance, the accumulation of the bacterial division protein FtsZ at the surface of droplets formed by complex coacervation of guanosine triphosphate (GTP) and polylysine was reported to induce extensive invaginations of the coacervate surface under mechanical pressure. ${ }^{144]}$ Shape instabilities and self-division were also reported in anisotropic liquid droplets formed by actin filaments. ${ }^{[145]}$ In another notable study, the dissipative self-assembly of FtsZ filaments was demonstrated in RNA/polypeptide complex coacervates in the presence of guanosine triphosphate ${ }^{[146]}$ (Figure 4E). Significantly, directional elongation of the coacervate droplets along the filaments, followed by their fission associated to the division of the FtsZ fibrils into two, was reported and rationalized using diffusion-reaction kinetics coupled to capillary forces.

Taken together, these studies demonstrate that energy-consuming, out-of-equilibrium processes may result in active fission of coacervate micro-droplets via localized cytoskeletal filament assembly. An experimental jump is needed to combine both active droplet growth and division to demonstrate complete duplication of LLPS-based protocells.

\section{Higher-order structures and behaviours}

\subsection{Internal droplet structuration and multi-phase organization}

Unlike synthetic LLPS, where the number and chemical nature of the components used are well defined, intracellular biomolecular condensation involves complex mixtures of various proteins and polynucleotides. This chemical diversity can produce vacuolization and multi-phase structuration within condensates in living cells, where multiple aqueous phases co-exist without mixing. ${ }^{[147]}$ This hierarchical organization may allow the spatial orchestration of multi-step reactions and has recently been observed in stress granules, ${ }^{[148]}$ nuclear speckles, ${ }^{[149]}$ and the nucleolus, ${ }^{[150]}$ where it has been attributed to balanced surface tensions between co-existing liquid phases ${ }^{[150]}$ and/or differences in effective solvation volumes. ${ }^{[151]}$ 
Reproducing such a multi-layered organisation in all-aqueous synthetic systems is very challenging due to the low interfacial energies involved. Dynamic substructures (vacuoles) have been observed within poly-Llysine/oligonucleotide coacervate micro-droplets subjected to a continuous electric field. [152] Dynamic vacuolization was also observed in RNA/peptide coacervate droplets produced via DNA transcription, and attributed to a reentrant phase transition process. ${ }^{[117,118]}$ Studies on ternary (or more) mixtures of phase-separating components are still relatively scarce. ${ }^{[153]}$ Multi-phase organization was recently achieved by addition of a PDDA/ATP coacervate bulk phase embedded to a PEG-suspended dextran-rich droplet, but this organization did not form spontaneously. ${ }^{[74]}$ In comparison, in a notable work, elastin-like polypeptides were designed to spontaneously produce complex organization upon thermally driven phase separation, including nano- or micro-droplets-withindroplet microstructures and multi-layered droplets. ${ }^{[154]}$ In another example, spatially organized droplets with subcompartments were reported in RNA-protein systems via the rational design of RNA sequences and modulation of the interactions involved between components. ${ }^{[155]}$ While much work remains to approach the structural complexity observed in biomolecular condensates, it is anticipated that future experimental studies, together with theoretical modelling, will help understanding and dynamically controlling multiphase organization in synthetic LLPS, paving the way to the regulation of biochemical reactions via the spatiotemporal control over the localization of biomolecules.

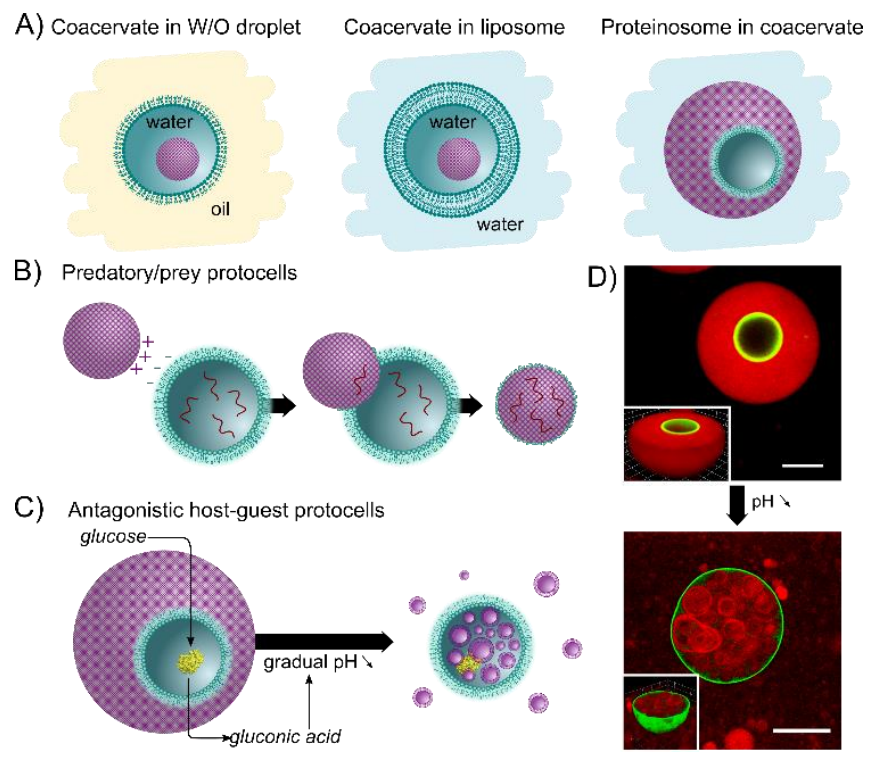

Figure 5. A) Scheme of examples of multi-compartmentalized structures involving LLPS-based droplets. B) Schematic representation of predatory/prey behaviour between protease-loaded coacervate droplets and proteinosomes (see ref. 163). C) Schematic representation of antagonistic chemical coupling in proteinosome-in-coacervate host-guest protocells, where the enzyme-mediated conversion of glucose into gluconic acid induces a pH decrease that transforms the fatty acid micelle coacervates into fatty acid vesicles. Some vesicles self-assemble within the proteinosomes to give a nested vesicle-in-proteinosome structure (see ref. 158). D) Confocal microscopy image of a single proteinosome-in-coacervate host-guest protocell (inset: 3D reconstruction) and their self-transformation into a vesicle-in-proteinosome constuct. Adapted from ref. 158 published under a CC BY 4.0 license.

\subsection{Multi-compartmentalization: towards artificial organelles}

The hierarchical, multi-compartmentalized structure of modern cells allows localization and positional assembly of related functional components and confinement of incompatible species, resulting in the spatiotemporal control over concerted multi-step reactions. The design of artificial organelles via the fabrication of multicompartmentalized synthetic cells has emerged in recent years as a promising approach to increase their structural and functional complexity (Figure 5A). To date, efforts have mainly focused on the construction of nested lipid or polymer vesicles, ${ }^{[156]}$ but multi-compartments involving LLPS have also recently been achieved. For instance, droplet-based microfluidics was used to produce monodisperse surfactant-based coacervates ${ }^{[34]}$ or PEGsuspended dextran-rich droplets[77] within water-in-oil droplets. Another study demonstrated the formation of protease-sensitive coacervate droplets water droplets suspended in mineral oil as a means to reproduce dynamic organelle assembly and dissolution in response to proteolytic degradation. ${ }^{[15]}$ This type or structure was recently extended to more relevant protocell models by Hück and co-workers who demonstrated the microfluidic-assisted fabrication of giant unilamellar vesicles encapsulating a single coacervate micro-droplet. ${ }^{[157]}$ Interestingly, droplets formed by complex coacervation between RNA and spermine exhibited a temperature-responsive behaviour, which allowed to dynamically control the storage and release of biomolecules, including cell-free protein expression machinery.

The first report to achieve the inverse nested architecture (synthetic membrane-bound compartment-incoacervate) was from Martin et al. who demonstrated the spontaneous capture and sequestration of protein-based microcapsules (proteinosomes) within fatty acid micelles coacervate droplets. ${ }^{[158]}$ Significantly, the formation of these multi-compartments did not require any complex processing techniques but simply relied on the passive 
wetting of proteinosomes by the coacervate micro-droplets. This spontaneous multi-compartmentalization strategy has also been used to capture biological organelles (chloroplasts) within complex coacervate micro-droplets based on complimentary charge interactions, resulting in photosynthetically active protocells capable of light energy capture and conversion. ${ }^{[159]}$ Taken together, these studies show that the capture and sequestration of membranebound compartments (synthetic or natural) within liquid-like droplets formed by LLPS may be a general approach for the spontaneous assembly of new multi-compartmentalized ensembles that could have had implications in the endocytotic formation of the first multi-compartmentalized cells.

\subsection{LLPS-based synthetic cell populations}

An emerging paradigm in the field of synthetic cells is the construction of larger ensembles of micro-compartments, referred to as communities of synthetic cells. ${ }^{[160]}$ Synthetic cells in a community may be related to each other via physical interactions and/or chemical coupling. Interestingly, when networked to each other, synthetic microcompartments within communities exhibit population-level phenomena and collective behaviours that may not be seen in individual objects. Recent examples based on membrane-bound compartments include the phagocytosisinspired engulfment of microcapsules by magnetically responsive colloidosomes, ${ }^{[161]}$ or the chemical binding of multiple proteinosomes together to form beating synthetic prototissues. ${ }^{[162]}$

The use of aqueous micro-droplets formed by LLPS at the population-level is an emerging perspective in the field of synthetic cells. Two directions are being explored. In the first approach, micro-droplets are immobilized (e.g. on a surface), then exposed to a diffusing chemical signal to which they respond. For instance, Tian et al. reported dynamic chemical sensing in an acoustically patterned two-dimensional array of enzymatically-active coacervate droplets, and further achieved chemical coupling between two coacervate droplets populations based on a concerted enzyme cascade reaction. ${ }^{[125]}$ In the second approach, micro-droplets remain in suspension where they diffuse freely and interact with other synthetic cells, via either physical interactions or chemical coupling. In a pioneering example, Qiao et al. reported a binary population of predatory/prey protocells ${ }^{[163]}$ (Figure 5B). Complex coacervate droplets loaded with protease enzymes (predatory protocells) were shown to physically bind to proteinosomes (preys) via complimentary charge interactions, resulting in the proteolysis of the proteinosomes' membrane and in the capture of the proteinosomes' content. In another notable work, Martin et al. demonstrated antagonistic chemical coupling in nested "host-guest" protocells as a primitive form of artificial parasitism [158] (Figures 5C,D). Here, the production of gluconic acid by glucose oxidase encapsulated in proteinosomes ("guest" protocells) resulted in the programmed disassembly of fatty acid micelle coacervate droplets ("host" protocells) and their transformation into fatty acid vesicles. Significantly, some vesicles were transferred to the proteinosomes, along with cargo biomolecules sequestered in the coacervate droplets, resulting in a new nested vesicle-inproteinosome structure. Overall, these recent examples of community-level behaviour in LLPS-based synthetic cells demonstrate how collective properties emerge from the physical or chemical interactions between heterogeneous compartments, and may inspire the design of new colloidal microsystems capable of life-inspired, dynamic behaviours, such as artificial predation, parasitism or symbiosis.

\section{Summary and outlook}

Liquid-liquid phase separation processes in water produce membrane-free droplets that are emerging as viable models to assemble dynamic synthetic cells, capable of molecular exchange, biochemical activity, structural transformations, reversible assembly and dissolution, and growth and division. However, the relatively simple physicochemical approaches used to create dynamic droplets still lie far away from the exquisite complexity observed in living cells. The ultimate goal would be to assemble dissipative systems, where self-organization across multiple length- and time-scales would be achieved via competing reactions that would synthesize and degrade matter at the same time. Recent progress in the fields of synthetic biology and systems chemistry suggest that it is now time for a conceptual and experimental advance. The integration of complex enzyme networks, cell-free expression systems, or gene-mediated circuits, together with molecular replication, dissipative adaptation, energydriven self-assembly, and new approaches based on population-level phenomena, should fuel the construction of self-sustained and self-regulated synthetic cells with emergent properties. Ultimately, beyond opening perspectives for the design of life-inspired artificial materials able to tackle key societal challenges, the combination of dynamic LLPS-based compartmentalization, bioactivity, molecular replication, dissipative self-assembly and collective behaviours will mark a stepping-stone towards our understanding of what life is.

\section{Acknowledgements}

NM acknowledges funding from IdEx Bordeaux (ANR-10-IDEX-03-02), an Investissement d'Avenir program of the French government managed by the Agence Nationale de la Recherche; and thanks Jean-Christophe Baret and Jean-Paul Douliez for helpful discussions.

Keywords: synthetic cells $\bullet$ liquid-liquid phase separation $\bullet$ compartmentalization $\bullet$ dynamic organization $\bullet$ microdroplets 
[2] Y. Dou, K. Dhatt-Gauthier, K.J.M. Bishop, Curr. Opin. Solid St. M. 2019, in press

[3] S. Mann, Acc. Chem. Res. 2012, 45, 2131-2141.

[4] A.A. Hyman, C.A. Weber, F. Jülicher, Annu. Rev. Cell. Dev. Biol. 2014, 30, 39-58.

[5] R.J. Wheeler, A.A. Hyman, Phil. Trans. R. Soc. B 2018, 373, 20170193.

[6] K. Bergfeldt, L. Picullel, P. Linse, J. Phys. Chem. 1996, 100, 3680-3687.

[7] P.A. Albertsson, in Partition of Cell Particles and Macromolecules, 3rd ed.; Wiley-Interscience: New York, 1986.

[8] a) A. Gustafsson, H. Wennerstrom, Polymer 1986, 27, 1768-1770; b) H.O. Johansson, G. Karlstrom, F. Tjerneld, C.A. Haynes, J. Chromatogr. B Biomed. Sci. Appl. 1998, 711, 3-17; c) T. Furuya, Y. Iwai, Y. Tanaka, H. Uchida, S. Yamada, Y. Arai Fluid Phase Equilib. 1995, $103,119-141$.

[9] B.Y. Zaslavsky, in Aqueous two-phase partitioning: physical chemistry and bioanalytical applications. Edited by B.Y. Zaslavsky, Marcel Dekker, Inc.; New York, 1994

[10] Y. Liu, R. Lipowsky, R. Dimova Langmuir 2012, 28, 3831-3839.

[11] G. Johansson, J. Chromatogr. 1978, 150, 63-71.

[12] C.D. Keating, Acc. Chem. Res. 2012, 45, 2114-2124.

[13] M.R. Helfrich, L.K. Mangeney-Slavin, M.S. Long, K.Y. Djoko, C.D. Keating, J. Am. Chem. Soc. 2002, 124, 13374-13375.

[14] M.S. Long, C.D. Jones, M.R. Helfrich, L.K., Mangeney-Slavin, C.D. Keating, Proc. Natl. Acad. Sci. 2005, 102, 5920-5925.

[15] M.S. Long, A.S. Cans, C.D. Keating, J. Am. Chem. Soc. 2008, 130, 756-762.

[16] a) H.B. de Jong, H.R. Kruyt, Proc. K. Ned. Akad. Wet. 1929, 32, 849-856; b) H.G. Bungenberg de Jong, in Colloid Science, Vol. 2 (Ed.: H.R. Kruyt), Elsevier: New York, 1949

[17] a) C.G. de Kruif, F. Weinbreck, R. de Vries Curr. Opin. Colloid. Int. Sci. 2004, 9, 340-349; b) C.L. Cooper, P.L. Dubin, A.B. Kayitmazer, S. Turksen, Curr. Opin. Colloid. Int. Sci. 2005, 10, 52-78; c) S.L. Turgeon, C. Schmitt, C. Sanchez, Curr. Opin. Colloid. Int. Sci. 2007, 12, 166-178; d) T. Croguennec, G.M. Tavares, S. Bouhallab, Adv. Colloid Int. Sci. 2017, 239, 115-126; e) L. Faltova, A.M. Küffner, M. Hondele, K. Weis, P. Arosio, ACS Nano 2018, 12, 9991-9999.

[18] T.Y.D. Tang, M. Antognozzi, J.A. Vicary, A.W. Perriman, S. Mann, Soft Matter 2013, 9, 7647-7646.

[19] J.R. Vieregg, M. Lueckheide, A.B. Marciel, L. Leon, A.J. Bologna, J. Reyes Rivera, M.V. Tirrell J. Am. Chem. Soc. 2018, 140, 1632-1638.

[20] S. Koga, D.S. Williams, A.W. Perriman, S. Mann, Nat. Chem. 2011, 3, 720-724

[21] a) M. Wang, Y. Wang, Soft Matter 2014, 10, 7909-7919; b) W. Zhao, Y. Wang, Adv. Colloid Int. Sci. 2017, 239, 199-212.

[22] a) Sing, C.E. Adv. Colloid. Int. Sci. 2016, 239, 2-16; b) M. Castelnovo, J.F. Joanny, Eur. Phys. J. 2001, 1, 203-214 ; c) A. Kudlay, A.V. Ermoshkin, M.O. de la Cruz, Macromolecules 2004, 37, 9213-9241; d) X. Liu, J.P. Chapel, C. Schatz, Adv. Colloid. Int. Sci. 2017, 239, $178-186$.

[23] Q. Wang, J.B. Schlenoff, Macromolecules 2014, 47, 3108-3116.

[24] a) S.L. Perry, Curr. Opin. Coll. Int. Sci. 2019, accepted manuscript; b) W.C. Blocher, S.L. Perry, WIREs Nanomed. Nanobio. 2017, 9, e1442.

[25] E. Spruijt, A.H. Westphal, J.W. Borst, M.A. Cohen Stuart, J. van der Gucht Macromolecules 2010, 43, 6476-6484.

[26] L.W. Chang, T.K. Lytle, M. Radhakrishna, J.J. Madinya, J. Vélez, C.E. Sing, S.L. Perry, Nat. Commun. 2017, 8, 1273.

[27] a) S.L. Perry, L. Leon, K.Q. Hoffmann, M.J. Kade, D. Priftis, K.A. Black, D. Wong, R.A. Klein, C.F. Pierce III, K.P. Margossian, J.K. Whitmer, J. Qin, J.J. de Pablo, M. Tirrell, Nat. Commun. 2015, 6, 6052; b) D. Priftis, L. Leon, Z. Song, S.L. Perry, K.O. Margossian, A. Tropnikova, J. Cheng, M. Tirrell, Angew. Chem. Int. Ed. 2015, 54, 11128-11132.

[28] A. Shakya, J.T. King, Biophys. J. 2018, 115, 1840-1847.

[29] B.J. Jeon, D.T. Nguyen, G.R. Abraham, N. Conrad, D.K. Fygenson, O.A. Saleh, Soft Matter 2018, 14, 7009-7015.

[30] a) E. Spruijt, M.A. Cohen Stuart, J. van der Gucht, Macromolecules, 2013, 46, 1633-1641; b) Y. Liu, H.H. Winter, S.L. Perry, Adv. Colloid. Int. Sci. 2017, 239, 46-60; c) Y.S. Jho, H.Y. Yoo, Y. Lin, S. Han, D.S. Hwang, Adv. Colloid. Int. Sci. 2017, 239, 61-73; d) E. Spruijt, J. Sprakel, M.A. Cohen Stuart, J. van der Gucht, Soft Matter 2010, 6, 172.

[31] A.I. Oparin, in Origin of Life. New York: Macmillan; 1938

[32] A.I. Oparin, K.L. Gladilin, Biosystems 1980, 12, 133-145.

[33] D. Garenne, L. Béven, L. Navailles, F. Nallet, E.J. Dufourc, J.P. Douliez, Angew. Chem. Int. Ed. 2016, 55, 13475-13479.

[34] J.P. Douliez, N. Martin, C. Gaillard, T. Beneyton, J.C. Baret, S. Mann, L. Béven, Angew. Chem. Int. Ed. 2017, 56, 13689-13693.

[35] a) C.P. Brangwynne, C.R. Eckmann, D.S. Courson, A. Rybarska, C. Hoege, J. Gharakhani, F. Jülicher, A.A. Hyman, Science, 2009, 324, 1729 1732; b) C.P. Brangwynne, J. Cell. Biol. 2013, 203, 875-881.

[36] E.W. Martin, T. Mittag, Biochemistry 2018, 57, 2478-2487.

[37] C.P. Brangwynne, P. Tompa, R.V. Pappu, Nat. Phys. 2015, 11, 899-904

[38] D.M. Mitrea, B. Chandra, M.C. Ferrolino, E.B. Gibbs, M. Tolbert, M.R. White, R.W. Kriwacki, J. Mol. Biol. 2018, 430, 4773-4805.

[39] J. Berry, C.P. Brangwynne, M. Haataja, Rep. Prog. Phys. 2018, 81, 046601.

[40] A.S. Holehouse, R.V. Pappu, Biochemistry 2018, 57, 2415-2423.

[41] S. An, R. Kumar, E.D. Sheets, S.J. Benkovic, Science 2008, 320, 103-106.

[42] A. Kumar Rai, J.X. Chen, M. Selbach, L. Pelkmans, Nature 2018, 559, 211-216.

[43] T. Stoetger, N. Battich, L. Pelkmans, Cell 2016, 164, 1151-1161.

[44] a) Y. Shin, C.P. Brangwynne, Science 2017, 357, eaaf4382; b) J.B. Woodruff, A.A. Hyman, E. Boke, Trends Biocehm. Sci. 2018 , $43,81-94$.

[45] P. Gruner, B. Riechers, B. Semin, J. Lim, A. Johnston, K. Short, J.C. Baret, Nat. Commun. 2016, 7, 10392.

[46] D.S. Williams, S. Koga, C.R.C. Hak, A. Majrekar, A.J. Patil, A.W. Perriman, S. Mann, Soft Matter 2012, 8, 6004-6014.

[47] W.M. Aumiller Jr, C.D. Keating, Adv. Colloid Int. Sci. 2017, 239, 75-87.

[48] a) R.R. Poudyal, F. Pir Cakmak, C.D. Keating, P.C. Bevilacqua, Biochemistry 2018, 57, 2509-2519; b) J.R. Vieregg, T.Y.D. Tang, Curr. Opin. Colloid. Int. Sci. 2016, 26, 50-57; c) C.D. Crowe, C.D. Keating, Interface Focus 2018, 8, 20180032.

[49] E.A. Frankel, P.C. Bevilacqua, C.D. Keating, Langmuir 2016, 32, 2041-2049.

[50] C.A., Strulson, R.C. Molden, C.D. Keating, P.C. Bevilacqua, Nat. Chem. 2012, 4, 941-946.

[51] N. Nakatani, H. Sakuta, M. Hayashi, S. Tanaka, K. Takiguchi, K. Tsumoto, K. Yoshikawa, ChemBioChem 2018, $19,1370-1374$.

[52] T.Z. Jia, C. Hentrich, J.W. Szostak, Orig. Life Evol. Biosph. 2014, 44, 1-12.

[53] D. Van Swaay, T.Y.D. Tang, S. Mann, A. de Mello, Angew. Chem. Int. Ed. 2015, 54, 8398-8401.

[54] E.M. Langdon, Y. Qiu, A.G. Niaki, G.A. McLaughlin, C.A. Weidmann, T.M. Gerbich, J.A. Smith, J.M. Crutchley, C.M. Termini, K.M. Weeks, S. Myong, A.S. Gladfelter, Science 2018, 360, 922-927.

[55] B. Drobot, J.M. Iglesias-Artola, K. Le Vay, V. Mayr, M. Kar, M. Kreysing, H. Mutschler, T.Y.D. Tang, Nat. Commun. 2018, 9 , 3643.

[56] T.J. Nott, T.D. Craggs, A.J. Baldwin, Nat. Chem. 2016, 8, 569-575.

[57] a) H. Di Nucci, B. Nerli, G. Pico, Biophys. Chem. 2001, 89, 219-229; b) L.M., Dominak, E.L., Gundermann, C.D. Keating, Langmuir 2010, 26, 56975705.

[58] N. Martin, M. Li, S. Mann, Langmuir 2016, 32, 5881-5889.

[59] M. Zhao, N.S. Zacharia, J. Chem. Phys. 2018, 149, 163326. 
[60] S. Lindhoud, M.M.A.E. Claessens, Soft Matter 2016, 12, 408-413.

[61] K.A. Black, D. Priftis, S.L. Perry, J. Yip, W.Y. Byun, M. Tirrell, ACS Macro Lett. 2014, 3, 1088-1091.

[62] S.F. Banani, A.M. Rice, W.B. Peeples, Y. Lin, S. Jain, R. Parker, M.K. Rosen, Cell 2016, 166, $651-663$.

[63] A.B. Kayitmazer, D. Seeman, B.B. Minsky, P.L. Dubin, Y. Xu, Soft Matter 2013, 9, 2553 -2583

[64] A.C. Obermeyer, C.E. Mills, X.H. Dong, R.J. Flores, B.D. Olsen, Soft Matter 2016, 12, 3570-3581.

[65] R.A. Kapelner, A.C. Obermeyer, Chem. Sci. 2019, 10, 2700-2707.

[66] A. Shakya, J.T. King, ACS Macro Lett. 2018, 7, 1220-1225.

[67] W. Stroberg, S. Schnell, Biophys. J. 2018, 115, 3-8.

[68] R.R. Poudyal, R.M. Guth-Metzler, A.J. Veenis, E.A. Frankel, C.D. Keating, P.C. Bevilacqua, Nat. Commun. 2019, $10,490$.

[69] G.F. Joyce, Nature 2002, 418, 214-221.

[70] a) T.P. Fraccia, G. Zanchetta, V. Rimoldi, N.A. Clark, T. Bellini, Origins Life Evol. B. 2015, 45, 51-68; b) T.P. Fraccia, G.P. Smith, G. Zanchetta, E. Paraboschi, Y. Yi, D.M. Walba, G. Dieci, N.A. Clark, T. Bellini, Nat. Commun. 2015, 6, 6424; c) S. Di Leo, M. Todisco, T. Bellini, T.P. Fraccia, Liq. Cryst. 2018, 45, 2306-2318; d) M. Todisco, T.P. Fraccia, G.P. Smith, A. Corno, L. Bethge, S. Klussmann, E.M. Paraboschi, R. Asselta, D. Colombo, G. Zanchetta, N.A. Clark, T. Bellini, ACS Nano 2018, 12, 9750-9762.

[71] a) P. Torre, C.D. Keating, S.S. Mansy, Langmuir 2014, 30, 5695-5699; b) E. Sokolova, E. Spruijt, M.M.K. Hansen, E. Dubuc, J. Groen, V. Chokkalingam, A. Piruska, H.A. Heuse, W.T.S. Huck, Proc. Natl. Acad. Sci. 2013, 110, 11692-11697; c) T.Y.D. Tang, D. van Swaay, A. deMello, J.L.R. Anderson, S. Mann, Chem. Commun. 2015, 51, 11429-11432.

[72] J. Crosby, T. Treadwell, M. Hammerton, K. Vasilakis, M.P. Crump, D.S. Williams, S. Mann, Chem. Commun. 2012, 48, 11832-11834.

[73] B.W. Davis, W.M. Aumiller, N. Hashemian, S. An, A. Armaou, C.D. Keating, Biophys. J. 2015, $109,2182-2194$.

[74] T. Kojima, S. Takayama, ACS Appl. Mater. Interfaces 2018, 10, 32782-32791.

[75] T. Nicolai, B. Murray, Food Hydrocolloids 2017, 68, 157-163.

[76] a) G. Balakrishnan, T. Nicolai, L. Benyahia L., D. Durand Langmuir 2012, 28, 5921-5926; b) J. Zhang, J. Hwang, M. Antonietti, B.V.K.J. Schmidt, Biomacromolecules 2019, 20, 204-211, c) A.T. Poortinga, Langmuir 2008, 24, 1644-1647.

[77] J.P. Douliez, N. Martin, T. Beneyton, J.C. Eloi, J.P. Chapel, L. Navailles, J.C. Baret, S. Mann, L. Béven, Angew. Chem. Int. Ed. 2018, 57, 77807784

[78] D.C. Dewey, C.A. Strulson, D.N. Cacace, P.C. Bevilacqua, C.D. Keating, Nat. Commun. 2014, 5, 4670

[79] D.N. Cacace, A.T. Rowland, J.J. Stapleton, D.C. Dewey, C.D. Keating, Langmuir, 2015, 31, 11329-11338.

[80] a) B.T. Nguyen, T. Nicolai, L. Benyahia, Langmuir 2013, 29, 10658-10664; b) A. Gonzalez-Jordan, T. Nicolai, L. Benyahia, Langmuir 2016, 32, 7189-7197; c) L.H. Xue, C.Y. Xie, S.X. Meng, R.X. Bai, X. Yang, Y. Wang, S. Wang, B.P. Binks, T. Guo, T. Meng, ACS Macro Lett. 2017, 6, 679 683.

[81] F. Pir-Cakmak, C.D. Keating, Sci. Rep. 2015, 7, 3215.

[82] K.R. Peddireddy, T. Nicolai, L. Benyahia, I. Capron ACS Macro Lett. 2016, 5, 283-286.

[83] Y. Song, U. Shimanovich, T.C.T. Michaels, Q. Ma, J. Li, T.P.J. Knowles, H.C. Shum Nat. Commun. 2016, 7, 12934

[84] B.P. Binks, Langmuir 2017, 33, 6947-6963.

[85] W.M. Aumiller Jr, F. Pir Cakmak, B.W. Davis, C.D. Keating, Langmuir 2016, 32, 10042-10053.

[86] J. Fothergill, M. Li, S.A. Davis, J.A. Cunnigham, S. Mann, Langmuir 2014, 30, 14591-14596.

[87] T.Y.D. Tang, C.R. Che Hak, A.J. Thomson, M.K. Kuimova, D.S. Williams, A.W. Perriman, S. Mann, Nat. Chem. 2014, 6, 527-533.

[88] D.M.A. Buzza, P.D.I. Fletcher, T.K. Georgiou, N. Ghasdian, Langmuir 2013, 29, 14804-14814.

[89] A.F. Mason, B.C. Buddingh', D.S. Williams, J.C.M. van Hest, J. Am. Chem. Soc. 2017, 139, $17309-17312$. <https://pubs.acs.org/doi/10.1021/jacs.7b10846>

[90] D.S. Williams, A.J. Patil, S. Mann, Small 2014, 10, 1830-1840.

[91] K. Nakai, U. Kwolek, J. Bednar, M. Zatorska, M. Nowakowska, M. Kepczynski, S. Yusa Eur. Polym. J. 2017, 94, 125-135.

[92] A. Ianiro, H. Wu, M.M.J. van Rijt, M.P. Vena, A.D.A Keizer, A.C.C. Esteves, R. Tuinier, H. Friedrich, N.A.J.M. Sommerdijk, J.P. Patterson, Nat. Chem. 2019, in press. https://doi.org/10.1038/s41557-019-0210-4

[93] a) D. Priftis, M. Tirrell, Soft Matter 2012, 8, 9396-9405, b) S.L. Perry, Y. Li, D. Priftis, L. Leon, M. Tirrell, Polymers 2014, 6, 1756-1772.

[94] P.L Onuchic, A.N. Milin, I.S.M. Alshareedah, A.A. Deniz, P.R. Banerjee, bioRxiv, 2019, doi: https://doi.org/10.1101/453019

[95] a) K. Kaibara, T. Okazaki, H.B. Bohidar, P.L. Dubin, Biomacromolecules 2000, 1, 100-107; b) P.J. Kha, P.S. Desai, J. Li, R.G. Larson, Polymers 2014, 6, 1414-1436.

[96] a) C.H. Eskiw, G. Dellaire, J.S. Mymryk, D.P. Bazzett-Jones, J. Cell Sci. 2003, 116, 4455-4466; b) E.W.J. Wallace, J.L. Kear-Scott, E.V. Pilipenko, M.H. Schwartz, P.R. Laskowski, A.E. Rojek, C.D. Katanski, J.A. Riback, M.F. Dion, A.M. Franks, E.M. Airoldi, T. Pan, B.A. Budnik, A. Drummond, Cell 2015, 162, 1286-1298.

[97] T.J. Nott, E. Petsalaki, P. Farber, D. Jervis, E. Fussner, A. Plochowietz, T.D. Craggs, D.P. Bazett-Jones, T. Pawson, J.D. Forman-Kay, A.J. Balwin, Mol. Cell 2015, 57, 936-947.

[98] H. Jiang, S. Wang, Y. Huang, X. He, H. Cui, X. Zhu, Y. Zheng, Cell 2015, 163, 108-122.

[99] D. Roy, W.L.A. Brooks, B.S. Sumerlin, Chem. Soc. Rev. 2013, 42, 7214-7243.

[100] a) D.W. Urry, B. Starcher, S.M. Partridge, Nature 1969, 222, 795-796; b) K.M. Ruff, S. Roberts, A. Chilkoti, R.V. Pappu, J. Mol. Biol. 2018, 430, 4619-4635.

[101] a) M. Amiram, F. Garcia Quiroz, D.J. Callahan, A. Chilkoti, Nat. Mater. 2011, 10, 141-148 ; b) J.R. Mcdaniel, D.C. Radford, A. Chilkoti, Biomacromolecules 2013, 14, 2866-2872; c) F. Garcia Quiroz, A. Chilkoti, Nat. Mater. 2015, 14, 1164-1171.

[102] J.R. Simon, N.J. Carroll, M. Rubinstein, A. Chilkoti, G.P. Lopez, Nat. Chem. 2017, 6, 509-515.

[103] R. Mérindol, S. Loescher, A. Samanta, A. Walther, Nat. Nanotech. 2018, 13, 730-738.

[104] a) Y. Shin, J. Berry, N. Pannucci, M.P. Haataja, J.E. Toettcher, C.P. Brangwynne, Cell 2017, 168, 159-171; b) D. Bracha, M.T. Walls, M.T. Wei, L. Zhu, M. Kurian, J.L. Avalos, J.E. Toettcher, C.P. Branwynne, Cell 2018, 175, 1467-1480.

[105] J. Edahiro, K. Sumaru, T. Takagi, T. Shinbo, T. Kanamori, Langmuir 2006, 22, 5224-5226.

[106] a) J.T. Wang, J. Smith, B.C. Cheng, H. Schmidt, D. Rasoloson, A. Paix, B.G. Lambrus, D. Calidas, A. Betzig, G. Seydoux, eLife 2014, 3, e04591; b) F. Wippich, B. Bodenmiller, M. Gustafsson Trajkovska, S. Wanka, R. Aebersold, L. Pelkmans, Cell 2013, $152,791-805$.

[107] Z. Monahan, V.H. Ryan, A.M. Janke, K.A. Burke, S.N. Rhoads, G.H. Zerze, R. O’Meally, G.L. Dignon, A.E. Conicella, W. Zheng, R.B. Best, R.N. Cole, J. Mittal, F. Shewmaker, N.L. Fawzi, EMBO J. 2017, 36, 2951-2967.

[108] A. Kumar Rai, J.X. Chen, M. Selbach, L. Pelkmans, Nature 2018, 559, 211-216.

[109] W.M. Aumiller Jr, C.D. Keating, Nat. Chem. 2016, 8, 129-137.

[110] K.K. Nakashima, J.F. Baaji, E. Spruijt, Soft Matter 2018, 14, 361-367.

[111] S. Yang, B. Li, C. Wu, W. Xu, M. Tu, Y. Yan, J. Huang, M. Dreschler, S. Granick, L. Jiang, ACS Nano, 2019, 13, $2420-2426$.

[112] S.C. Weber, C.P. Brangwynne, Curr. Biol. 2015, 25, 641-646. 
[113] N.N. Deng, M.A. Vibhute, L. Zheng, H. Zhao, M. Yelleswarapu, W.T.S. Huck, J. Am. Chem. Soc. 2018, 140, 7399-7402.

[114] S. Mytnyk, A.G.L. Olive, F. Versluis, J.M. Poolman, E. Mendes, R. Eelkema, J.H. van Esch, Angew. Chem. Int. Ed. $2017,56,14923-14927$.

[115] B.S. Schuster, E.H. Reed, R. Parthasarathy, C.N. Jahnke, R.M. Caldwell, J.G. Bermudez, H. Ramage, M.C. Good, D.A. Hammer, Nat. Commun. 2018, 9, 2985.

[116] S.N. Semenov, A.S.Y. Wong, R.M. van der Made, S.G.J. Postma, J. Groen, H.W.H. van Roekel, T.F.A. de Greef, W.T.S. Huck, Nat. Chem. 2015, 7, 160-165.

[117] P.R. Banerjee, A.N. Milin, M.M. Moosa, P.L. Onuchic, A.A. Deniz, Angew. Chem. Int. Ed. 2017, 56, 11354-11359.

[118] A.N. Milin, A.A. Deniz, Biochem. 2018, 57, 2470-2477.

[119] Y. Caspi, C. Dekker, Syst. Synth. Biol. 2014, 8, 249-269.

[120] I. Ivanov, R.B. Lira, T.Y.D. Tang, T. Franzmann, A. Klosin, L. Caire da Silva, A.A. Hyman, K. Landfester, R. Lipowsky, K. Sundmacher, R. Dimova, Adv. Biosyst. 2019, 1800314, earyl view https://doi.org/10.1002/adbi.201800314

[121] M.M. Hanczyc, S.M. Fujikawa, J.W. Szostak, Science 2003, 302, 618-622.

[122] T.F. Zhu, J.W. Szostak, J. Am. Chem. Soc. 2009, 131, 5705-5713.

[123] J. Berry, S.C. Weber, N. Vaidya, M. Haataja, C.P. Brangwynne, Proc. Natl. Acad. Sci. 2015, 112, E5237-E5245.

[124] L. Tian, N. Martin, P.G. Bassindale, A.J. Patil, M. Li, A. Barnes, B.W. Drinkwater, S. Mann, Nat. Commun. 2016, 7, 13068.

[125] L. Tian, M. Li, J. Liu, A.J. Patil, B.W. Drinkwater, S. Mann, ACS Cent. Sci. 2018, 4, 1551-1558.

[126] D. Zwicker, R. Seyboldt, C.A. Weber, A.A. Hyman, F. Jülicher, Nat. Phys. 2017, 13, 408-413.

[127] a) J.W. Szostak, D.P. Bartel, P.L Luisi, Nature 2013, 409, 387-390 ; b) G. Ashkenasy, T.M. Hermans, S. Otto, A.F. Taylor, Chem. Soc. Rev. 2017, 46, 2443-2554 ; c) K. Ruiz-Mirazo, C. Briones, A. de la Escosura, Chem. Rev. 2014, 114, 285-366.

[128] J.W. Taylor, S.A. Eghtesadi, L.J. Points, T. Liu, L. Cronin, Nat. Commun. 2017, 8, 237.

[129] Y. Song, T.C.T. Michaels, Q. Ma, Z. Liu, H. Yuan, S. Takayama, T.P.J. Knowles, H.C. Shum, Nat. Commun. 2018, 9, 2110.

[130] M. Andes-Koback, C.D. Keating, J. Am. Chem. Soc. 2011, 133, 9545-9555.

[131] R. Dimova, R. Lipowsky, Adv. Mater. Interfaces 2017, 4, 1600451.

[132] Y. Li, H. Kusumaatmaja, R. Lipowsky, R. Dimova J. Phys. Chem. B 2012, 116, 1819-1823.

[133] R. Lipowsky, J. Phys. Chem. B 2018, 122, 3572-3586.

[134] a) S. Banjade, M.K. Rosen, eLife, 2014, 3, e04123; b) P.A. Chong, J.D. Forman-Kay, Curr. Opin. Struct. Biol. 2016, 41, $180-186$.

[135] M. Zeng, Y. Shang, Y. Araki, T. Guo, R.L. Huganir, M. Zhang, Cell 2016, 166, 1163-1175.

[136] a) M.M. Lacy, R. Ma, N.G. Ravindra, J. Berro, FEBS Lett. 2018, 595, 3586-3605; b) L.P. Bergeron-Sandoval, H.K. Heris, A.G. Hendricks, A.J. Ehrlicher, P. François, R.V. Pappu, S.W. Michnick, biorXiv 2018; c) L.P. Bergeron-Sandoval, S.W. Michnick, J. Mol. Biol. 2018, 430, 4754-4761

[137] N. Martin, K.P. Sharma, R.L. Harniman, R.M. Richardson, R.J. Hutchings, D. Alibhai, M. Li, S. Mann, Sci. Rep. $2017,7,41327$.

[138] R. Seybolt, F. Jülicher, New J. Phys. 2018, 20, 105010.

[139] a) K.P. Browne, D.A. Walekr, K.J.M. Bishop, B.A. Grzybowski, Angew. Chem. Int. Ed. 2010, 49, 6756-6759; b) F. Caschera, S. Rasmussen, M.M. Hanczyc, ChemPlusChem 2012, 78, 52-54 ; c) I. Derenyi, I. Lagzi, Phys. Chem. Chem. Phys. 2014, 16, 4639-4641; d) Y. Sato, K. Yasuhara, J.I. Kikuchi, T.N. Sato, Sci. Rep. 2013, 3, 3475; e) J. Cejkova, T. Banno, M.M. Hanczyc, F. Stepanek, Art. Life 2017, $23,528-549$.

[140] a) D. Merkkle, N. Kahya, P. Schwille, ChemBioCHem 2008, 9, 2673-2681; b) G. Rivas, S.K. Vogel, P. Schwille, Curr. Opin. Chem. Biol. 2014, 22, $18-26$; c) M. Jimenez, A. Martos, A.J. Cabré, A. Raso, G. Rivas, Environ. Microbiol. 2013, 15, 3158-3168.

[141] a) B. Monterroso, S. Zorrilla, M. Sobrinos-Sanguino, C.D. Keating, G. Rivas, Sci. Rep. 2016, 6, 35140 ; b) M. Sobrinos-Sanguino, S. Zorrilla, C.D. Keating, B. Monterroso, G. Rivas, Chem. Commun. 2017, 53, 4775-4778.

[142] B. Monterroso, S. Zorrilla, M. Sobrinos-Sanguino, M.A. Robles-Ramos, M. Lopez-Alvarez, C.D. Keating, G. Rivas, biorXiv 2018, doi: https://doi.org/10.1101/264192

[143] P.M. McCall, S. Srivastava, S.L. Perry, D.R. Kovar, M.L. Gardel, M.V. Tirrell, Biophys. J. 2018, 114, $1636-1645$.

[144] F. Fanalista, S. Deshpande, A. Lau, G. Pawlik, C. Dekker, Adv. Biophys. 2018, 2, 1800-136.

[145] a) K.L. Weirich, S. Banerjee, K. Dasbiswas, T.A. Witten, S. Vaikuntanathan, M.L. Gardel, Proc. Natl. Acad. Sci. 2017, 114, 2131-2136; b) K.L. Weirich, K. Dasbiswas, T.A. Witten, S. Vaikuntanathan, M.L. Gardel, bioRxiv, 2018, doi: https://doi.org/10.1101/403121

[146] E. te Brinke, J. Groen, A. Herrmann, H.A. Heus, G. Rivas, E. Spruijt, W.T.S. Huck, Nat. Nanotech. $2018,13,849-855$.

[147] a) H. Broder Schmidt, R. Rohatgi, Cell Rep. 2016, 16, 1228-1236; b) K. Galsior, J. Zhao, G. McLaughin, M.G. Forest, A.S. Gladfelter, J. Newby, Phys. Rev. E 2019, 99, 012411; c) I.A. Sawyer, D. Sturgill, M. Dundr, WIREs RNA 2019, 10, c1514.

[148] S. Jain, J.R. Wheeler, R.W. Walters, A. Agrawal, A. Barsic, R. Parker, Cell 2018, 164, 487-498.

[149] J. Fei, M. Jadaliha, T.S. Harmon, I.T.S. Li, B. Hua, Q. Hao, A.S. Holehouse, M. Reyer, Q. Sun, S.M. Freier, R.V. Pappu, K.V. Pasanth, T. Ha, J. Cell Sci. 2017, 130, 4180-4192.

[150] M. Feric, N. Vaidya, T.S. Harmon, D.M. Mitrea, L. Zhu, T.M. Richardson, R.W. Kriwacki, R.V. Pappu, C.P. Brangwynne, Cell 2016, 165, 16861697.

[151] T.S. Harmon, A.S. Holehouse, R.V. Pappu, New J. Phys. 2018, 20, 045002.

[152] a) Y. Tin, X. Zhu, M. Zhao, Z. Zhang, S. Mann, D. Liang, Nat. Commun 2016, 7, 10658; b) Y. Yin, H. Chang, H. Jing, Z. Zhang, D. Yan, S. Mann, D. Liang, Soft Matter 2018, 14, 6514-6520.

[153] a) D. Priftis, X. Xia, K.O. Margossian, S.L. Perry, L. Leon, J. Qin, J.J. de Pablo, M. Tirrell, Macromolecules 2014, 47, 3076-3085; b) M. Zhao, X. Xia, J. Mao, C. Wang, M.B. Dawadi, D.A. Modarelli, N.S. Zacharia, Mol. Syst. Des. Engin. 2019, 4, 110-121.

[154] J.R. Simon, N.J. Carroll, M. Rubinstein, A. Chilkoti, G.P. Lopez, Nat. Chem. 2017, 9, 509-515.

[155] S. Boeynaems, A.S. Holehouse, V. Weinhardt, D. Kovacs, J. Van Lindt, C. Larabell, L. Van Den Bosch, R. Das, P.S. Tompa, R.V. Pappu, A.D. Gitler, bioRxiv, 2018, doi: https://doi.org/10.1101/492793

[156] a) M. Marguet, C. Bonduelle, S. Lecommandoux, Chem. Soc. Rev. 2013, 42, 512-529 ; b) T. Trantidou, M.S. Friddin, A. Saleh-Reyhani, O. Ces, Y. Elani, Lab Chip 2018, 18, 2488-2509 ; c) K. Göpfrich, I. Platzman, J.P. Spatz, Trends Biotechnol. 2018, 36, 938-951.

[157] N.N. Deng, W.T.S. Huck, Angew. Chem. Int. Ed. 2017, 56, 9736-9740.

[158] N. Martin, J.P. Douliez, Y. Qiao, R. Booth, M. Li, S. Mann, Nat. Commun. 2018, 9, 3652.

[159] B.V.V.S.P. Kumar, J. Fothergill, J. Bretherton, L. Tian, A.J. Patil, S.A. Davis, S. Mann, Chem. Commun. $2018,54,3594-3597$.

[160] P. Stano, Life, 2019, 9, 3

[161] L. Rodriguez-Arco, M. Li, S. Mann, Nat. Mater. 2017, 16, 857-863.

[162] P. Gobbo, A.J. Patil, M. Li, R. Harniman, W.H. Briscoe, S. Mann, Nat. Mater. 2018, 17, 1145-1153.

[163] Y. Qiao, R. Booth, M. Li, S. Mann, Nat. Chem. 2017, 9, 110-119. 